\title{
« Optimal production of transplant care services »
}

\author{
$\underline{\text { Auteurs }}$ \\ Bruno Deffains, Jean Mercier Ythier \\ Document de Travail n 2009 - 19
}

Mai 2009

\section{Faculté des sciences économiques et de gestion}

Pôle européen de gestion et d'économie (PEGE) 61 avenue de la Forêt Noire F-67085 Strasbourg Cedex

Secétariat du BETA Géraldine Manderscheidt Tél. : (33) 0390242069 Fax : (33) 0390242070 g.manderscheidt@unistra.fr http://cournot2.u-strasbg.fr/beta 


\title{
Optimal production of transplant care services
}

\author{
Bruno Deffains \\ Université de Paris Ouest-Nanterre, and CNRS (EconomiX) \\ Jean Mercier Ythier \\ Université de Metz, CNRS (BETA) and IDEP
}

December, 2008

\begin{abstract}
Most organ transplants are from dead donors. National transplant organizations exhibit considerable differences in terms of their donor population rates. Spain's organization is by far the most efficient in this respect. We argue that much of the productivity advantage of Spain's transplant organization proceeds from an efficient organization of the production chain, from organ procurement to transplantation. Transplant inputs from dead donors are analogous to a common resource for the transplant community. Their circulation through the national transplant organization creates public good externalities between the care units in charge of organ extraction and those in charge of transplantation. It is shown that a socially efficient production of transplant services requires an optimal control of both the production and the circulation of transplant inputs by the institutions of the transplant system.
\end{abstract}

Keywords: organ transplants; donation; public goods; production organization

JEL codes: H41, H51, H57, I12, I18, K11, K23, L23, L38, L53

\section{Introduction}

The demand for life-saving transplant surgery grows in most of countries. However, the organ donor rates as well as the total number of transplants differ tremendously from one country to another. During the last ten years, Spain appears as the champion of the dead donor league. Considering that $90 \%$ of organ transplants, in general, are coming from dead donors ${ }^{1}$, it is crucial to understand the reasons of such a success. In practice, the Spanish transplant system improves survival, it increases organ demand, and more and more people are taking advantage from transplantation. For many commentators, these achievements clearly show that organ donation is the limiting factor to treat certain pathologies. This is certainly partly true, but still insufficient to explain the differences, in terms of production efficiency, of transplant care systems around the world. We argue in this article that the problem is not only, and perhaps not mainly, with the lack of donors per se, but, rather, with the organization of the transplant system, and notably of its production side.

\footnotetext{
${ }^{1}$ IRODaT, 2006.
} 
A surprisingly small number of papers concentrate on organization aspects in the economic literature on organ transplants. Notable exceptions are the recent contributions of Roth, Sönmez and Ünver (2004, 2005ab, 2006). They consider the case of live kidney donations, and design theoretical patterns of gift-exchange for efficient pairwise matching of kidney donors and recipients from a given set of pairs of incompatible donor and recipient. They present numerical simulations of the impact of such discrete optimization procedures on transplant provision, and consider the practical implementation of these procedures by means of specialized clearinghouses (see notably Roth et alii (2005a) concerning the latter).

As recalled above, and acknowledged by these authors themselves (e.g. Roth et alii (2004)), most transplanted kidneys, as most transplanted organs in general, are from cadavers. Law commonly makes cadaveric transplant donation much easier than live transplant donation, notably by presuming the consent of deceased donors. For instance, in many countries, consent is taken for granted in principle if the brain-dead patient has not expressed her/his refusal explicitly before death through some relevant legal procedure (usually, his/her registration on an ad hoc legal file). The medical staff in charge of organ procurement is generally submitted to an obligation to check the wishes of the deceased regarding donation, by interviewing close relative. Moreover, refusal rates usually are much lower than 50\% in organ donations interviews: $15 \%$ in Spain, but also 27\% in France and 39\% in the UK for example. These facts point to the crucial importance, for the productive efficiency of the transplant care organization, of the latter's ability in identifying and exploiting opportunities of organ procurement from cadaveric donations.

This article develops an economic model of the transplant care system within existing legal environments, with a particular emphasis on the organization of the production side of the system. The ban of organs markets makes transplants a common resource, collected mainly by "exhortation", that is, notably, by public calls for donation (Thorne $(2000,2006))$. The bulk of the "resource" is constituted by brain-dead patients randomly distributed in hospitals through the statistical variety of death circumstances, and physically non-transferable for a variety of reasons that notably include the stringent legal obligations relative to the body of the deceased. This initial distribution of the common resource is naturally mismatched, in general, with the statistical distribution of the needs of grafts for 
transplantation in care production units. Operating an appropriate match of resources and "needs" in transplant inputs is the basic reason for the existence of institutions in charge of circulating grafts, such as national transplant agencies, as substitutes for banned transplant markets. Grafts are produced by hospitals, and circulated by the transplant agency, to be used by other hospitals as inputs in their final production of transplant care services. Hospital's intermediary graft production thus induces public good external effects on others' final production of care services. The resulting public good issues are captured through principal-agent interactions, in subgame-perfect equilibria of two-stage games where hospitals are only concerned with their own final production of care services while the transplant agency maximizes a social utility function that aggregates hospitals' preferences (Bergstrom (1989), Cornes and Silva (1999)). It is notably shown that: (i) a socially optimal control of the agency over both the circulation and the production of graft inputs achieves social optimum ; (ii) while a socially optimal control by the agency of circulation alone generally implies suboptimal underprovision of transplant inputs and services. We argue that the model so captures an essential cause of the remarkable achievements of the Spanish transplant organization.

The paper develops as follows. Section 2 analyzes the Spanish transplant organization. Section 3 presents the model of the transplant care system. Section 4 sets and solves the public good problem of graft production and circulation. Section 5 concludes. An appendix collects the proofs.

\section{Spain's transplant organization}

The history of organs transplantations in Spain begins in 1965, with the first transplants in Madrid and Barcelona. In 1979, a law is adopted to favour the development of transplantations but donations remain at a low level during the eighties.

In 1989, the Organizacion Nacional de Trasplantes (ONT) is created to solve this problem. It is an institution belonging to the Ministry of Health and Consumption, put in charge of developing the competencies relative to the provision and clinical utilization of organs and tissues. To carry out these tasks, it functions as a technical operative unit and fulfils its mission of coordinating the activities of donation, extraction, preservation, distribution, exchange, and transplantation of organs and tissues 
throughout the whole Spanish Health Care System. At the creation of the ONT, the main idea was that the problem was not with the number of donors but with their identification and the organization of the program.

After the creation of the ONT, Spain went from 14 donors per million population (pmp) in 1989 to 36,4 donors pmp in 2006. This evolution displayed in the graphic below made Spain evolve from donation rates ranked in intermediate-low positions in Europe to the highest rate not just in Europe, but also worldwide.

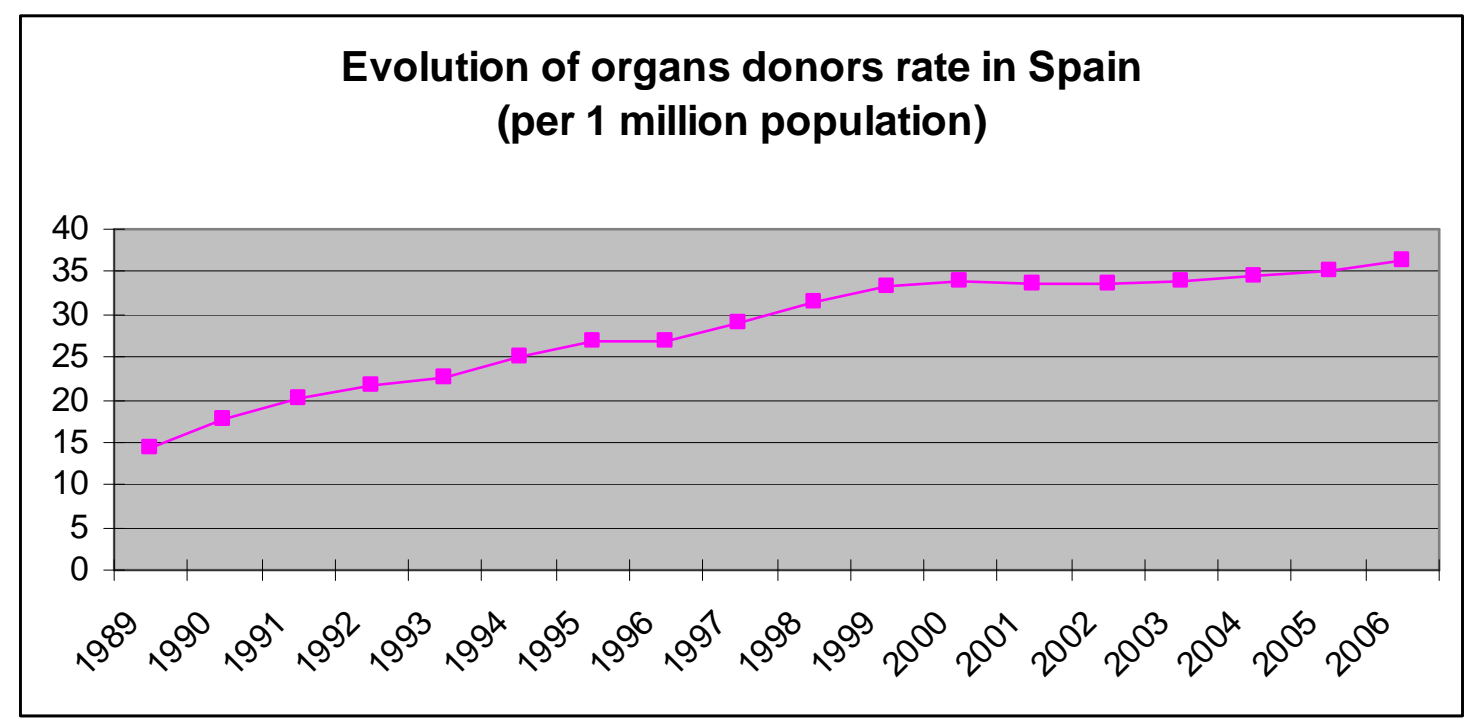

How has this been possible? The origin of this spectacular change is internationally known as the "Spanish Model", a series of measures taken in this country to improve organ donation. This model, widely described in the scientific literature, has been recommended by the World Health Organization and is being applied in different regions of the World with outcomes very similar to those obtained in Spain.

Spanish transplant law is very similar to the corresponding laws in other Western countries. Although the law on transplant donation presumes the consent of deceased potential donors, according to a subsequent decree relatives of a potential donor must be approached to determine the deceased's wishes regarding organ donation. In the absence of this knowledge, close relatives can sign the authorization, after internal discussion if required. At present, Spain's annual refusal rate for organ donation is around $15 \%$ of all donation interviews. Death is defined as the total and irreversible cessation of brain or cardio-respiratory functions. Clinical evaluation and complimentary tests required 
are detailed within the legal text allowing organ retrieval either from brain-stem death donors or from non-heart-beating donors. Like other coordinating systems worldwide, the Spanish system has to monitor the management of waiting lists, organ allocation, and statistical analysis. Nevertheless, it was considered that a continuous monitoring system over the entire organ donation process was essential. A network of health-care professionals responsible for the organ donation process as a whole has been set up at all levels (national, regional and hospital). This implies the need for training, organization and coordination of activities.

It was considered that these professionals working at the grass roots level must feel involved and that they must be accountable for performance. Most of them are physicians, mainly intensive care unit (ICU) specialists, and they belong to the staff of the hospital. They generally continue in their medical role, but as transplant coordinators their main objective is to improve the organ donation rate. Currently, 155 hospitals are officially authorized to take care of organ donor programs. A quality control system has been developed for the organ donor process - the ICU mortality registry and the brain death registry - a common practice in most of them. By law (RD 2070, 30 December 1999), transplant coordinators are the professionals responsible for the whole donation and retrieval process. National and regional offices are service agencies supporting the organ donation and transplantation programs. They deal with organ sharing and waiting list management. They arrange organ or team shifts. They are responsible for the official statistics and reports on organ donation and transplantation. They promote legal statements and binding consensus guidelines. They also promote public education and address any doubt or question about organ donation and transplantation. A 24-h hot line and Email system have been put in place to keep all interested groups or individuals informed. They are also concerned with and involved in training and research programs. Any activity that could improve donation or facilitate the transplant team activities can be promoted through this network.

Organ transplantation has been considered a hospital medical activity for which a specific budget and staff are allocated. This kind of activity does not induce any budgetary overload for hospitals. The annual general budget for transplantation procedures in Spain is around 180 million Euros. The annual budget for the organ procurement network is around 15 million Euros (less than 10\% of the budget covering organ procurement activities). The general donation budget covers all extra-salary and extra- 
time activities of both coordinators and surgical retrieval teams, as well as any donor evaluation tests, the ICU bed daily costs, etc. This budget also covers coordinating offices, training courses and some of the educational programs. The type of payment for the extra work of coordination and organ retrieval for professionals in charge differs depending on the region. It can be a fixed amount, or it can be based on registered activity, or be determined according to a mixed system (it does not usually exceed $30 \%$ of total salary).

Table 1 shows that the Spanish organ donor rate per million population is the highest around the world. The British rate is only 37,5\% of the Spanish rate and the French rate 69,5\%. We could compute in table 2 a rough estimate of the number of patients waiting for kidney transplantation that would obtain in several countries if they achieved the same donor rate as Spain.

Table 1: Organs Donor Rates per 1 million population in 2006

\begin{tabular}{|cc|}
\hline & 2006 \\
\hline Australia & 9 \\
\hline Canada & 14,8 \\
\hline France & 25,3 \\
\hline Greece & 5,8 \\
\hline Israel & 7,7 \\
\hline Italy & 20,9 \\
\hline Spain & 36,4 \\
\hline Sweden & 14,5 \\
\hline UK & 13 \\
\hline United States & 26,6 \\
\hline
\end{tabular}


Table 2: Projections of the 2006 Spanish rate on other countries

\begin{tabular}{|c|c|c|c|}
\hline & Cadaveric donors (1) & Kidneys transplants (1) & $\begin{array}{l}\text { Patients awaiting for a } \\
\text { transplant in } 2007 \text { (2) }\end{array}$ \\
\hline Australia & 202 & 330 & 1388 \\
\hline Australia* & 565 & 1334 & 343 \\
\hline Canada & 468 & 712 & 4195 \\
\hline Canada* & 1151 & 1751 & 1705 \\
\hline France & 1441 & 2352 & 6491 \\
\hline France $^{*}$ & 2073 & 3383 & 4511 \\
\hline Greece & 74 & 144 & 903 \\
\hline Greece* $^{*}$ & 464 & 903 & 144 \\
\hline Israel & 68 & 87 & 540 \\
\hline Israel* $^{*}$ & 321 & 411 & 114 \\
\hline Italy & 1239 & 2932 & 7096 \\
\hline Italy* & 2157 & 5106 & 4074 \\
\hline UK & 633 & 1240 & 6876 \\
\hline$U K^{*}$ & 1772 & 3472 & 3472 \\
\hline United States & 8022 & 10659 & 76313 \\
\hline United States* & 10909 & 14496 & 55767 \\
\hline
\end{tabular}

(*)Numbers in italic are calculated using the national rates of Table 1

(1) Source: IRODaT 2006

(2) Source: Council of Europe, Transplant Newsletter, September 2008

To sum up, the Spanish model consists in a program designed to optimize every stages of the transplantation process from the identification of a potential donor. Many factors contribute to the 
extraordinary increase of the Spanish dead-donor rate during the last 20 years. Of course, Spain was a pioneer of the opt-out system ${ }^{2}$, but its success mainly proceeds from an excellent network of organtransplant teams operating in hospitals, which routinely screen patients' records to identify donors, and impulse and coordinate the multiple tasks following donors' identification.

\section{3-A model of production of transplant care services}

The simple medical care system that we consider here is made of care production units, named hospitals, and a transplant agency in charge of collecting transplants produced from cadavers by hospitals, and of distributing them to transplant care units. The use of grafts by hospitals is constrained by the following two complementary rules: they must transfer to the transplant agency any graft they produce; and they must use for transplant care services any graft they receive from the transplant agency. We suppose, for simplicity, undifferentiated resources and needs in transplant inputs (say, a single medical indication for transplantation, such as kidney pathology, for example), and hospitals identical in all respects except their potential resources in graft inputs (their brain-dead patients, principally).

\section{3-1-Agents and commodities}

There are $n$ hospitals, $n \geq 2$, designated by an index $i$ running in $N=\{1, \ldots, n\}$. The transplant agency is denoted by index $i=0$.

We partition the set of care services provided by hospitals into two broad classes, namely: Care services requiring transplants of organs or tissues such as heart, kidney, liver, lung, skin, cornea, bone marrow etc.; and all other care services. We assume that the transplant care services of hospital $i$, on the one hand, and its other care services, on the other hand, are measurable by homogeneous

\footnotetext{
${ }^{2}$ In the opt-out donation system, consent is presumed for deceased donors unless she/he registered on an appropriate refusal file when alive. In most opt-out systems, the next of kin's approval is also required. Spain, France, Italy for instance presume consent. In the opt-in system of donation, on the contrary, those willing to give their organs upon death must sign up as donors. Countries with opt-out systems have high deceased-donor rates.
} 
continuous variables, respectively denoted by $x_{i}$ and $y_{i}$. Moreover, each hospital $i$ is susceptible to produce grafts from cadavers in homogeneous continuous quantity $z_{i}$. The final output of the medical care system in transplant care services (resp. other care services) is vector $x=\left(x_{1}, \ldots, x_{n}\right)$ (resp. $\left.y=\left(y_{1}, \ldots, y_{n}\right)\right)$. Its intermediary production of transplants is vector $z=\left(z_{1}, \ldots, z_{n}\right)$. We denote by $z_{n / i}$ the vector obtained from $z$ by deleting its ith-component $z_{i}$, and by $\left(z_{n / i}, z_{i}^{\prime}\right)$ the vector obtained from $z$ and $z^{\prime}$ by substituting $z_{i}^{\prime}$ for $z_{i}$ in $z$.

Likewise, we bunch the variable inputs of the production of care services in two broad types, also viewed as homogeneous continuous quantities, that is, for any hospital $i$ : Transplants, denoted by real variable $t_{i}$; and other inputs, labelled "general" inputs in the sequel, and denoted by real variables $v_{i}^{x}$ if they are used in the production of transplant care services, $v_{i}^{y}$ if they are used in the production of other (final) care services, and $v_{i}^{z}$ if they are used in the production of grafts. We let $v_{i}=\left(v_{i}^{x}, v_{i}^{y}, v_{i}^{z}\right)$, $v^{r}=\left(v_{1}^{r}, \ldots, v_{n}^{r}\right)$ for any $r \in\{x, y, z\}$, and $v=\left(v_{1}, \ldots, v_{n}\right)$.

We use the following notations for vectors of $\mathbb{R}^{n}, n \geq 1: e_{n}$ is the diagonal vector $(1, \ldots, 1)$ of $\mathbb{R}^{n}$; for any pair $\left(x, x^{\prime}\right)$ of vectors of $\mathbb{R}^{n}, x \geq x^{\prime}$ if $x_{i} \geq x_{i}{ }^{\prime}$ for all $i, x>x^{\prime}$ if $x \geq x^{\prime}$ and $x \neq x^{\prime}, x \gg x^{\prime}$ if $x_{i}>x_{i}{ }^{\prime}$ for all $i ; \mathbb{R}_{+}^{n}$ is the non-negative orthant of $\mathbb{R}^{n}$, that is, set $\left\{x \in \mathbb{R}^{n}: x \geq 0\right\}$, and $\mathbb{R}_{++}^{n}$ is its positive orthant $\left\{x \in \mathbb{R}^{n}: x \gg 0\right\}$.

\section{3-2-Feasibility conditions}

Hospitals' potential of graft production is mainly determined, in practice, by the random distribution of brain-dead patients in hospitals and by refusal rates in donation interviews. This essential feature of the reality of transplant activities, which may be appropriately construed as a set of operative rationing constraints over both graft production and transplant care services, is captured in the model notably through an exogenous endowment of potential graft production of the hospital, viewed as a non- 
negative homogeneous continuous quantity, and denoted by $\omega_{i}$ for hospital $i .^{3}$ This endowment operates as an upper bound for hospital's graft production. We let $\omega=\left(\omega_{1}, \ldots, \omega_{n}\right)$, and suppose that $\omega \gg 0$.

Technically efficient production of hospital $i$ is depicted through a triple of production functions $f_{i}=\left(f_{i}^{x}, f_{i}^{y}, f_{i}^{z}\right)$ transforming nonnegative combinations of inputs $\left(t_{i}, v_{i}\right) \in \mathbb{R}_{+}^{4}$ into technically efficient output combinations $\left(x_{i}, y_{i}, z_{i}\right)=\left(f_{i}^{x}\left(t_{i}, v_{i}\right), f_{i}^{y}\left(t_{i}, v_{i}\right), f_{i}^{z}\left(t_{i}, v_{i}\right)\right)$. The formal assumption below supposes, in addition to the standard working hypotheses of differentiability and concavity, the following main features for hospitals' identical production techniques. General inputs are indispensable for production of any type (assumption 1-(ii)), and are productive in each type of production taken separately (assumptions 1-(iv) and 1-(v)) and also in the three types of production taken jointly (assumption 1-(vi)). Transplants are indispensable and productive in transplant care services (assumptions 1-(iii)-(iv)), and in them only (assumption 1-(v)). Technology exhibits a crowding externality between the three types of activities of each hospital (transplant care services, other care services, and graft production), specified as follows: Increasing the scale of production in terms of total general inputs in a hospital diminishes the productivity of general inputs in all types of production of this hospital, due to the crowding of a number of fixed inputs implicit in the production function, such as wards, operating theatres, surgery teams etc. (assumptions 1-(iv) and 1-(v)). Finally,

\footnotetext{
${ }^{3}$ Refusal rates in donation interviews, in particular, are treated as exogenous in this model, the latter's object being the analysis of the efficiency of production organization, from the extraction of donated organs to transplantation. Diminishing refusal rates and improving the organization of production are the two main channels for improving the global efficiency of transplant care systems as measured by their donor population rates. The first channel supposes appropriate exhortation policies, which may include an adequate management of donation interviews (see Thorne (1996, 2006:5.1) for an empirical estimation of the productivity of exhortation spending). Spain's low refusal rate accounts for a part of its high relative performance in terms of the donor rate, but seemingly not for the main part of it. Comparing, for example, the refusal and donor rates of France and Spain, one can produce estimates of the relative contributions of exhortation policy (say, the "exhortation effect") and production organization (say, the "organization of production effect") to the productivity gap between these two countries quite simply as follows: Substituting the French refusal rate (27\%) for the Spanish one (15\%) in Spanish donation data yields a Spanish donor rate net of the difference in exhortation policies of $36.4 \times \frac{1-0.27}{1-0.15}=31.26$ per million; the latter implies relative contributions of the exhortation effect and the organization of production effect to the productivity gap that are respectively of $\frac{36.4-31.26}{36.4-25.3}=46.3 \%$ and $\frac{31.26-25.3}{36.4-25.3}=53.7 \%$. Similar calculations conducted on UK data yield similar conclusions, namely, an exhortation effect and a production organization effect respectively accounting for $44 \%$ and $56 \%$ of the productivity gap between Spain and the UK.
} 
the marginal productivity of general inputs in any type of production exceeds their marginal selfcrowding impact (assumptions 1-(iv) and 1-(v)).

Assumption 1: (i) For all $r \in\{x, y, z\}, f_{i}^{r}$ is of the type $\left(t_{i}, v_{i}\right) \rightarrow g_{i}^{r}\left(t_{i}, v_{i}^{r}, v_{i}^{x}+v_{i}^{y}+v_{i}^{z}\right)$, where $g_{i}^{r}$ is continuous and concave in $\mathbb{R}_{+}^{3}$ and $C^{2}$ in $\mathbb{R}_{++}^{3}$. (ii) $g_{i}^{r}\left(t_{i}, v_{i}^{r}, v_{i}^{x}+v_{i}^{y}+v_{i}^{z}\right)=0$ whenever $v_{i}^{r}=0$. (iii) $g_{i}^{x}\left(t_{i}, v_{i}^{x}, v_{i}^{x}+v_{i}^{y}+v_{i}^{z}\right)=0$ whenever $t_{i}=0$. (iv) $g_{i}^{x}$ is $>0$, increasing in $t_{i}$, totally increasing in $v_{i}^{x}$, and is decreasing in total general input $v_{i}^{x}+v_{i}^{y}+v_{i}^{z}$ in $\mathbb{R}_{++}^{3}$ (that is, precisely: $g_{i}^{x}>0, \partial_{1} g_{i}^{x}>0$, $\partial_{2} g_{i}^{x}+\partial_{3} g_{i}^{x}>0$ and $\partial_{3} g_{i}^{x}<0$ in $\mathbb{R}_{++}^{3}$, where $\partial_{k} g_{i}^{x}$ denotes the partial derivative of $g_{i}^{x}$ with respect to its k-th argument, $k \in\{1,2,3\}$ ). (v) For all $r \in\{y, z\}, g_{i}^{r}$ is everywhere constant in $t_{i}$; it is $>0, C^{2}$, totally increasing in $v_{i}^{r}$, and decreasing in $v_{i}^{x}+v_{i}^{y}+v_{i}^{z}$ in $\mathbb{R}_{+} \times \mathbb{R}_{++}^{2}$ (i.e., with the notations above: $\partial_{1} g_{i}^{r}=0 ; \quad g_{i}^{r}>0, \partial_{2} g_{i}^{r}+\partial_{3} g_{i}^{r}>0$ and $\partial_{3} g_{i}^{r}<0$ in $\mathbb{R}_{+} \times \mathbb{R}_{++}^{2}$ ). (vi) For all $\left(t_{i}, v_{i}\right) \in \mathbb{R}_{+}^{3}$ and all neighbourhood $V$ of $\left(t_{i}, v_{i}\right)$ in $\mathbb{R}_{+}^{3}$, there exists $\tilde{v}_{i} \in \mathbb{R}_{+}^{2}$ such that $\left(t_{i}, \tilde{v}_{i}\right) \in V$ and $g^{r}\left(t_{i}, \tilde{v}_{i}, \tilde{v}_{i}^{x}+\tilde{v}_{i}^{y}+\tilde{v}_{i}^{z}\right)>g^{r}\left(t_{i}, v_{i}, v_{i}^{x}+v_{i}^{y}+v_{i}^{z}\right)$ for all $r \in\{x, y, z\}$. (vii) Hospitals' production constraints are identical, except for the upper bound on graft production, that is, there exists a triple of functions $\left(g^{x}, g^{y}, g^{z}\right)$ such that, for all $i:\left(g_{i}^{x}, g_{i}^{y}, g_{i}^{z}\right)=\left(g^{x}, g^{y}, g^{z}\right)$.

Assumption 1 is maintained throughout in the sequel.

Hospitals can purchase any quantity of general inputs $v_{i}^{x}+v_{i}^{y}+v_{i}^{z}$ on perfectly competitive markets of inputs at fixed market price $w$. Graft provision is non-profit: It is billed at production cost to the transplant agency, which collects transplants and redistributes them to care units free of charge. Each hospital $i$ finances its general inputs for care services from a fixed budget $B$, the same for all $i$, subject to budget constraint $w\left(v_{i}^{x}+v_{i}^{y}\right) \leq B$. The latter imposes an upper bound $B / w$ on its aggregate consumption of general inputs for care services $v_{i}^{x}+v_{i}^{y}$. The market price of general inputs is normalized to 1 in the sequel, that is, we let $w=1$, without loss of generality. 
The set of feasible alternatives of hospital $i$ that deduces from the assumptions above reads: $A_{i}\left(t_{i}, \omega_{i}\right)=\left\{\left(x_{i}, y_{i}, z_{i}, v_{i}\right) \in \mathbb{R}_{+}^{6}:\left(x_{i}, y_{i}, z_{i}\right) \leq g\left(t_{i}, v_{i}\right), z_{i} \leq \omega_{i}\right.$, and $\left.v_{i}^{x}+v_{i}^{y} \leq B\right\}$, where $g$ denotes map $\left(t_{i}, v_{i}\right) \rightarrow\left(g^{x}\left(t_{i}, v_{i}^{x}, v_{i}^{x}+v_{i}^{y}+v_{i}^{z}\right), g^{y}\left(t_{i}, v_{i}^{y}, v_{i}^{x}+v_{i}^{y}+v_{i}^{z}\right), g^{z}\left(t_{i}, v_{i}^{z}, v_{i}^{x}+v_{i}^{y}+v_{i}^{z}\right)\right)$.

The transplant agency is endowed with fixed budget $B_{0}$, sufficient to cover the cost of graft production for any feasible $z$, that is, $B_{0} \geq \sum_{i \in N} v_{i}^{z}$ for all $v$ such that $g^{z}\left(t_{i}, v_{i}\right) \leq \omega_{i}$ for all $i$. Its set of feasible alternatives therefore reads: $A_{0}(z)=\left\{t=\left(t_{1}, \ldots, t_{n}\right) \in \mathbb{R}_{+}^{n}: \sum_{i \in N} t_{i} \leq \sum_{i \in N} z_{i}\right\}$. This assumption notably implies, realistically enough we believe, that the rationing constraints over organ transplantation are entirely driven by technical and endowment limitations: They owe nothing in this model, and owe very little in practice, to the financial constraints of the medical care systems of developed economies.

\section{3-3-Hospital's production possibility frontier}

All relevant characteristics of hospitals' constraints can be conveniently summarized in the following notion of a production possibility frontier of the hospital, describing the set of hospital's accessible and technically efficient output combinations $\left(x_{i}, y_{i}, z_{i}\right)$. The formal definition below uses two functions derived in the first lemma of the appendix (see Appendix: A-1), namely: function $z_{i} \rightarrow\left(g_{B}^{z}\right)^{-1}\left(z_{i}\right)$, which yields the quantity of general inputs required to produce $z_{i}$ for any fixed total quantity of general inputs available for transplant and other care services $v_{i}^{x}+v_{i}^{y}=B$; and function $\left(x_{i}, z_{i}, t_{i}\right) \rightarrow F\left(x_{i}, z_{i}, t_{i}\right)$, which yields the efficient production in general care services $y_{i}$ accessible from any fixed accessible $\left(x_{i}, z_{i}\right)$ and any fixed positive $t_{i}$.

Definition 1: The production possibility frontier of hospital $i$ is: set $\left\{\left(x_{i}, y_{i}, z_{i}\right) \in \mathbb{R}_{+}^{3}: x_{i}=0, y_{i}=g^{y}\left(0, B, B+\left(g_{B}^{z}\right)^{-1}\left(z_{i}\right)\right)\right.$ and $\left.z_{i} \leq \omega_{i}\right\} \quad$ if $\quad t_{i}=0 ; \quad$ set $\left\{\left(x_{i}, y_{i}, z_{i}\right) \in \mathbb{R}_{+}^{3}: x_{i} \leq g^{x}\left(t_{i}, B, B+\left(g_{B}^{z}\right)^{-1}\left(z_{i}\right)\right), y_{i}=F\left(x_{i}, z_{i}, t_{i}\right)\right.$ and $\left.z_{i} \leq \omega_{i}\right\}$ if $t_{i}>0$. 
Figures $1 \mathrm{a}$ and $1 \mathrm{~b}$ represent the canonical projection of some production possibility frontier on plane $\left(x_{i}, y_{i}\right)$ for fixed pairs $\left(z_{i}, t_{i}\right)$ such that $t_{i}$ is respectively null and positive.

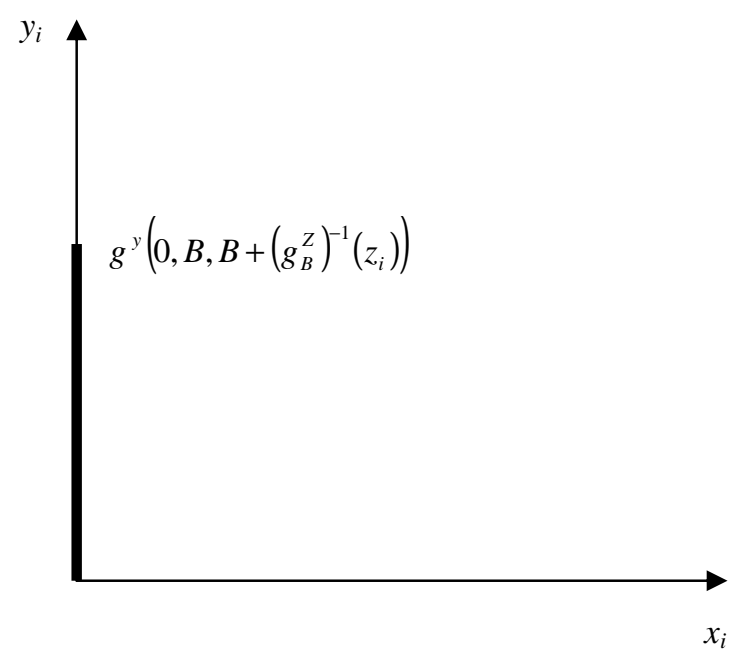

Fig 1.a

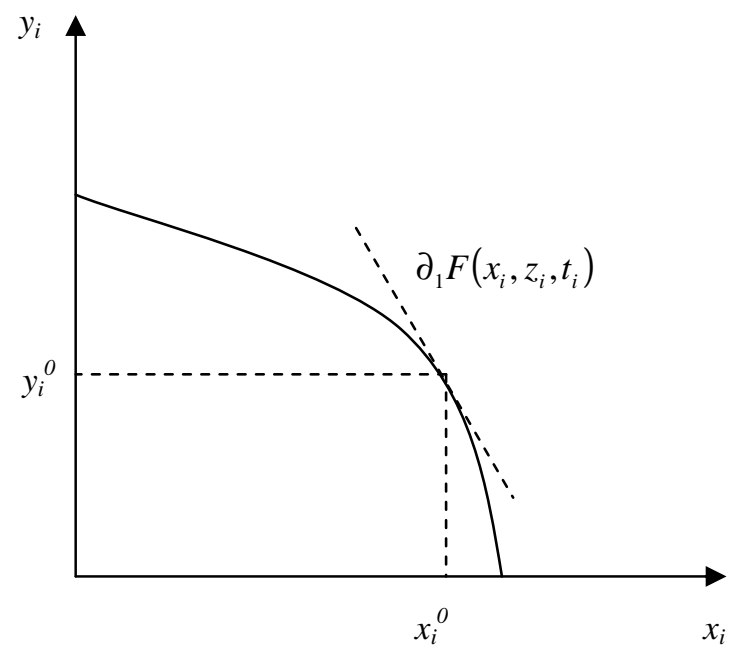

Fig 1.b

The partial derivative of function $F$ relative to $x_{i}$ calculated in Lemma 1 is $\partial_{1} F\left(x_{i}, z_{i}, t_{i}\right)=-\frac{\partial_{2} g^{y}\left(t_{i}, B-v_{i}^{x}, s\right)}{\partial_{2} g^{x}\left(t_{i}, v_{i}^{x}, s\right)}$, where $v_{i}^{x}$ is the quantity of general inputs used in transplant care (solving $x_{i}=g^{x}\left(t_{i}, v_{i}^{x}, s\right)$ ) and scale factor $s$ is the total consumption of general inputs 
$\left(=B+\left(g_{B}^{z}\right)^{-1}\left(z_{i}\right)\right)$. It interprets as a technical marginal rate of substitution of general care services for transplant care services, that is, the marginal variation (decrease) in the provision of general care services that is required for maintaining hospital's production combination on the production possibility frontier, following a marginal increase in the provision of transplant care services. It corresponds, geometrically, to the slope of the graph of partial function $x_{i} \rightarrow F\left(x_{i}, z_{i}, t_{i}\right)$ in plane $\left(x_{i}, y_{i}\right)$ (see Figure 1b). We name it marginal rate of transformation in the sequel, although it does not exactly coincide with usual meaning of the latter notion, to distinguish it from the marginal rate of substitution defined from hospitals' utility function below.

Another characteristic of hospital technology which reveals analytically indispensable in this model is the marginal rate of compensation of transplant provision by transplant transfer, formally defined as follows:

Definition 2: Let $t_{i}>0$, and $\left(x_{i}, y_{i}, z_{i}\right)$ be on hospital $i$ 's associate production possibility frontier. The marginal rate of compensation (MRC) of transplant provision by transplant transfer at $\left(x_{i}, y_{i}, z_{i}\right)$ is: $-\frac{\partial_{2} F\left(x_{i}, z_{i}, t_{i}\right)}{\partial_{3} F\left(x_{i}, z_{i}, t_{i}\right)}$.

Any increase in graft production $z_{i}$ ceteris paribus induces a downward shift of the graph of $x_{i} \rightarrow F\left(x_{i}, z_{i}, t_{i}\right)$ in plane $\left(x_{i}, y_{i}\right)$, implying a contraction of hospital's set of accessible production in $\left(x_{i}, y_{i}\right)$, due to the crowding effect exerted on the production of transplant and general care services by increased use of general inputs in graft production (see Lemma 1 and Figure 1c). Symmetrically, any increase in transplant transfer $t_{i}$ ceteris paribus induces an upward shift of the graph of $x_{i} \rightarrow F\left(x_{i}, z_{i}, t_{i}\right)$ in plane $\left(x_{i}, y_{i}\right)$ (implying an expansion of hospital's set of accessible final productions $\left.\left(x_{i}, y_{i}\right)\right)$ by releasing some quantity of general inputs in transplant care provision, which can be freely reallocated between $x_{i}$ and $y_{i}$ production without inducing any additional crowding of 
production capacities (Lemma 1 and Figure 1d). The marginal rate of compensation $-\frac{\partial_{2} F\left(x_{i}^{*}, z_{i}^{*}, t_{i}^{*}\right)}{\partial_{3} F\left(x_{i}^{*}, z_{i}^{*}, t_{i}^{*}\right)}$ measures the marginal variation (increase) in the transplant transfer $t_{i}^{*}(>0)$ received by hospital $i$ that is required for keeping hospital's production constant $\left(=F\left(x_{i}^{*}, z_{i}^{*}, t_{i}^{*}\right)\right)$, following a marginal increase in its graft production from $z_{i}^{*}$. It corresponds, geometrically, to the slope of the level curve through $\left(z_{i}^{*}, t_{i}^{*}\right)$ of partial function $\left(z_{i}, t_{i}\right) \rightarrow F\left(x_{i}^{*}, z_{i}, t_{i}\right)$ in plane $\left(z_{i}, t_{i}\right)$ (see Figure 1e). ${ }^{4}$ It is determined by the ratio of crowding costs to the marginal productivity of inputs, increasing in the former. ${ }^{5}$ It interprets as an indicator of tension on hospital's production capacities.

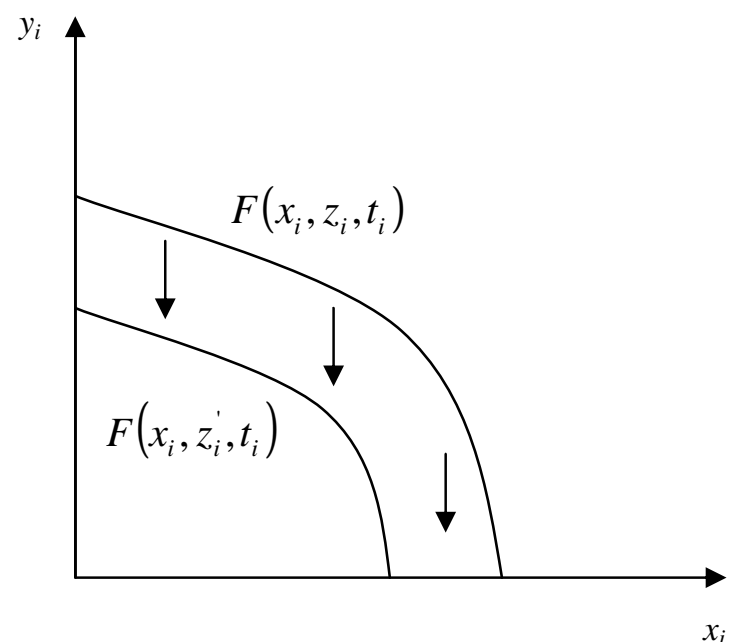

Fig 1.c

4 More formally, it follows from Lemma 1 and the implicit function theorem that equation $F\left(x_{i}, z_{i}, t_{i}\right)-F\left(x_{i}^{*}, z_{i}^{*}, t_{i}^{*}\right)=0$ implicitly defines $t_{i}$ as a $C^{2}$ increasing function $\left[0, \omega_{i}\right] \rightarrow \mathbb{R}_{++}$of $z_{i}$, the graph of which is the "level curve" of $\left(z_{i}, t_{i}\right) \rightarrow F\left(x_{i}^{*}, z_{i}, t_{i}\right)$ through $\left(z_{i}^{*}, t_{i}^{*}\right)$ in plane $\left(z_{i}, t_{i}\right)$. The implicit function theorem moreover implies that the first derivative of this implicit function is $=-\frac{\partial_{2} F\left(x_{i}^{*}, z_{i}, t_{i}\right)}{\partial_{3} F\left(x_{i}^{*}, z_{i}, t_{i}\right)}$ at any point $\left(z_{i}, t_{i}\right)$ of its graph.

${ }^{5}$ Calculations using the proof of Lemma 1 yield an MRC $=-\frac{1}{\partial_{2} g^{z}+\partial_{3} g^{z}}\left(\frac{\partial_{3} g^{x}}{\partial_{1} g^{x}}+\frac{\partial_{2} g^{x}}{\partial_{1} g^{x}} \cdot \frac{\partial_{3} g^{y}}{\partial_{2} g^{y}}\right)$, which is increasing in $\left|\partial_{3} g^{r}\right|$ for all $r \in\{x, y, z\}$. 


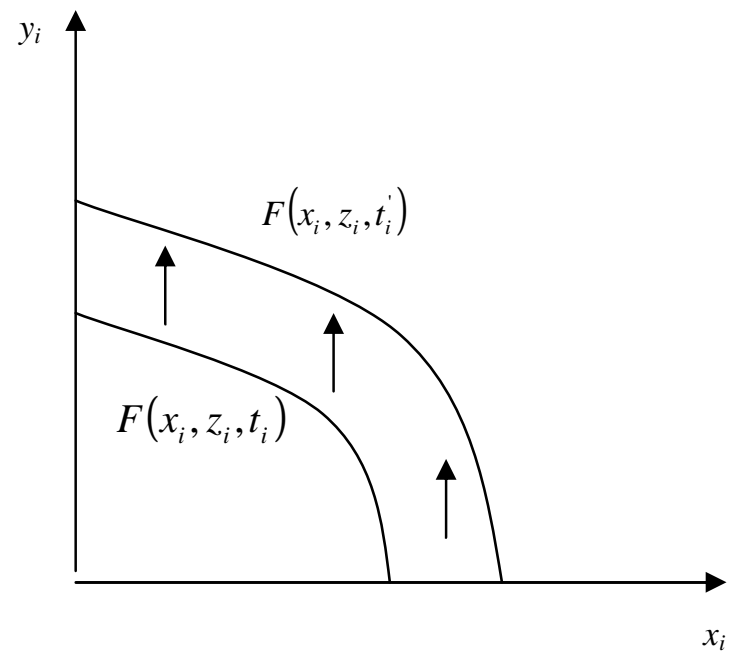

Fig 1.d

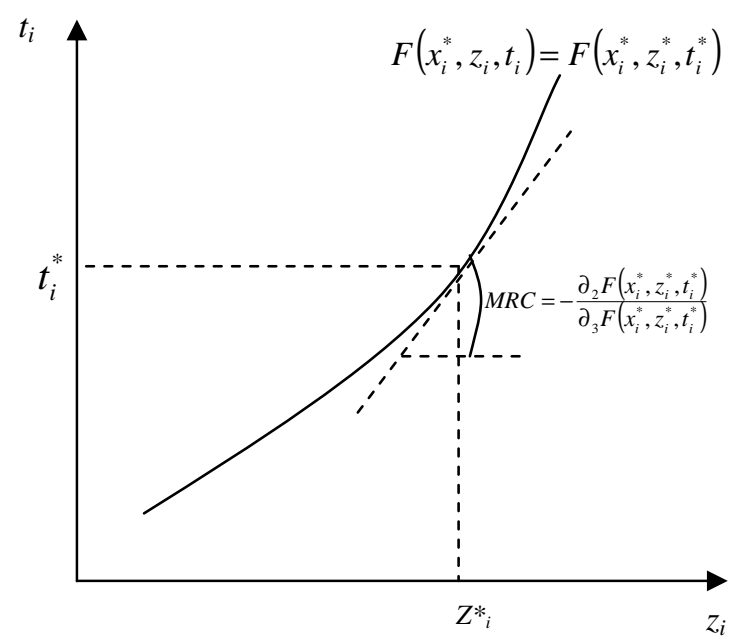

Fig 1.e

The position of the MRC relative to unity (larger than, equal to, or smaller than 1) is of particular importance for the study of equilibrium and social optimum below. To fix ideas, consider the case of a hospital that contributes an additional kidney for circulation by the transplant agency $\left(d z_{i}=+1\right)$ and simultaneously receives an additional kidney from the agency for its own transplant care services $\left(d t_{i}=+1\right)$. An MRC $>1($ resp. $=1$, resp. $<1)$ essentially means that its production possibility frontier shifts downwards (resp. is left unchanged, resp. shifts upwards) in the plane of final production combinations $\left(x_{i}, y_{i}\right)$ as a consequence of this marginal change in its pair of contribution and transfer $\left(z_{i}, t_{i}\right)$. 


\section{4-Behavioural assumptions: preferences, interactions and equilibrium}

In this section, we first return on one of the basic justifications for the existence of a transplant agency collecting and dispatching transplants (4-1). We turn next to the modelling of public good issues relative to transplants in the presence of the transplant agency (4-2-1 and 4-2-2), and finally design a socially optimal transplant care system (4-3).

\section{4-1-Unregulated equilibrium and the optimal production of transplant care services}

Our basic behavioural assumption is agent rationality, construed as the maximization of agents' complete and transitive preferences over their sets of alternatives.

Hospitals, notably, are viewed as rational agents exclusively concerned with their own provision of care services. Hospital $i$ maximizes a utility function $u$, the same for all $i$, over the set of pairs $\left(x_{i}, y_{i}\right)$ of combinations of transplant and other care services which it performs. We make the following set of standard assumptions on utility function, which will be maintained throughout the sequel:

Assumption 2: Hospitals' utility function is a continuous, non-decreasing, quasi-concave function over $\mathbb{R}_{+}^{2}$, whose restriction to $\mathbb{R}_{++}^{2}$ is a $C^{2}$, strictly increasing and strictly quasi-concave function. Moreover $u\left(x_{i}, y_{i}\right)>u(0)$ implies $\left(x_{i}, y_{i}\right) \gg 0 .^{6}$

\footnotetext{
${ }^{6}$ Note that the boundary condition of Assumption 2, which may interpret as a priority of the final production of care services, is incompatible with for-profit behaviour. Assumption 2 can be made compatible with for-profit behaviour for production plans that are sufficiently far from the lower boundary of hospital's production set by relaxing the strict quasi-concavity in $\mathbb{R}_{++}^{2}$ to simple quasi-concavity. Strict quasi-concavity is used in the sequel only for the technically convenient uniqueness of hospital's optimum final production plan that it implies. It can be replaced, with the same consequence and without substantial differences for subsequent analysis, by the alternative assumption of a quasi-concave utility function and production functions $g^{x}$ and $g^{y}$ strictly concave in $\mathbb{R}_{++}^{3}$. In other words, the main of subsequent analysis applies to the case of for-profit behaviour of care production units when equilibrium conditions imply an optimal production plan of the hospital that is sufficiently far from the axes.
} 
In the absence of a market for transplant inputs, banned by law, and of any institutional substitute for the former such as a transplant agency, hospitals would be reduced to a situation of autarky, as far as transplant inputs are concerned, that is, produce by themselves, from their own endowment $\omega_{i}$ and budget $B_{i}$, the grafts they use in their final production of transplant care services. Formally, each hospital would $\quad$ solve $\max \left\{u\left(x_{i}, y_{i}\right):\left(x_{i}, y_{i}, z_{i}, v_{i}, t_{i}\right) \geq 0\right.$, $\left(x_{i}, y_{i}, z_{i}\right) \leq g\left(t_{i}, v_{i}\right), t_{i} \leq z_{i} \leq \omega_{i}$, and $\left.v_{i}^{x}+v_{i}^{y}+v_{i}^{z} \leq B\right\}$, where hospital's budget covers all expenses in general inputs, including the general inputs $v_{i}^{z}$ used in intermediary graft production (hospital's "autarkic" budget constraint). A production equilibrium of this autarkic transplant care system would then consist of an input-output combination of the care system $(x, y, z, v, t)$ solving simultaneously the $n$ independent programs of the hospitals. We name this type of equilibrium an autarkic equilibrium.

Common sense suggests that such unregulated equilibrium can very easily result in the waste of a part of total graft resources, that is, typically, in this highly aggregated model ${ }^{7}$, disposal, by best endowed hospitals, of the fraction of their endowment that exceeds the quantity of graft inputs they need for the provision of transplant care services that maximizes their utility in program above. Formally:

Theorem 1: There exists a solution $\left(x_{i}^{*}, y_{i}^{*}, z_{i}^{*}, v_{i}^{*}, t_{i}^{*}\right)$ of the "unconstrained" autarkic program $\max \left\{u\left(x_{i}, y_{i}\right):\left(x_{i}, y_{i}, z_{i}, v_{i}, t_{i}\right) \geq 0,\left(x_{i}, y_{i}, z_{i}\right) \leq g\left(t_{i}, v_{i}\right), t_{i} \leq z_{i}\right.$, and $\left.v_{i}^{x}+v_{i}^{y}+v_{i}^{z} \leq B\right\}$, that is, of hospital's autarkic program where the rationing constraint over graft production $z_{i} \leq \omega_{i}$ has been dropped. This solution is the same for all $i$, unique with respect to $\left(x_{i}^{*}, y_{i}^{*}\right)$, and $\gg 0$. If there exists $\omega_{i}^{*} \in\left\{\omega_{i}: i \in N\right\}$ such that $\left(x_{i}^{*}, y_{i}^{*}, z_{i}^{*}, v_{i}^{*}, t_{i}^{*}\right)$ verifies rationing constraint $z_{i}<\omega_{i}^{*}$ with a strict inequality, then, for all hospitals $j$ such that $\omega_{j} \geq \omega_{i}^{*}:\left(x_{i}^{*}, y_{i}^{*}, z_{i}^{*}, v_{i}^{*}, t_{i}^{*}\right)$ is an autarkic equilibrium input-output combination of hospital $j$; and hospital $j$ 's marginal utility of graft resource $\omega_{i}^{*}$ is null

\footnotetext{
${ }^{7}$ In a more accurate description of the medical care system, the problem under consideration here would be, realistically, formulated as mismatched vectors of potential graft resources (kidneys, corneas,...) and final transplant care services of the hospital at any moment in time. For an application of matching models and discrete optimization techniques to the health care system and the economics of transplants, see Alvin Roth et alii $(2004,2005 \mathrm{ab}$ and 2006).
} 
at $\left(x_{i}^{*}, y_{i}^{*}, z_{i}^{*}, v_{i}^{*}, t_{i}^{*}\right)$. Moreover, hospitals' autarkic budget constraints $v_{i}^{x}+v_{i}^{y}+v_{i}^{z} \leq B$ are always binding (that is, satiated, with positive hospitals' marginal utilities of budget) at autarkic equilibrium.

The proof of this theorem, as those of most of the following, is detailed in the appendix (see A-2). Only one very short proof will be presented in the present section (Theorem 3).

The disposal of a part of the resources of the hospitals that are best endowed in terms of their potential of graft production will very commonly appear as a social waste if there exists a possibility of making a productive use of disposed resources in some other hospitals, in terms of their final production of care services. Hospitals' endowments in the sense above (potential of graft production) being physically and legally non-transferable, the notion of social optimum implicit in this normative appreciation of "wasteful" disposal actually refers to implicit social preferences over pairs $(x, y)$ of final production of the care system. ${ }^{8}$ We now introduce such preferences explicitly, with the following basic normative priors, summarized in Assumption 3 below: The social preferences aggregate hospitals' preferences, are increasing in both types of final production of care services, express (like hospitals') a priority of production, and imply a preference for an "equal treatment of relevantly equals", that is, a preference for equal provision of final care services over hospitals whenever the latter is accessible.

Assumption 3: The social utility function is a continuous, non-decreasing, anonymous ${ }^{9}$ function $W: \mathbb{R}_{+}^{n} \rightarrow \mathbb{R}_{+}$, whose restriction to $\mathbb{R}_{++}^{n}$ is a $C^{2}$, strictly increasing function $\mathbb{R}_{++}^{n} \rightarrow \mathbb{R}_{++}$. Moreover

\footnotetext{
${ }^{8}$ Brain-dead patients cannot be physically transferred from one hospital to another mainly because of imperative legal constraints. In particular, lump-sum transfers of hospitals' graft endowments cannot be used as instruments of a public distribution policy in this context. Grafts are physically transferable between hospitals, subject to the legal constraints of the national transplant organization, but they must be extracted on site, due to the reason above. Distribution $\omega$ cannot be an object of individual or social preference in our context (if preference underlies choice, as is assumed here, naturally). Graft production $z$ is individually or socially valuable only as an intermediary for the final production of transplant care services $x$. Final production of care services seems, therefore, to be the most appropriate object of preferences, both at individual and at social level, in this model of the medical care system.

${ }^{9}$ The anonymity property states that any permutation in hospitals' names (and associate production $\left(x_{i}, y_{i}\right)$ ) leaves the agency's utility unchanged.
} 
$(x, y) \rightarrow W\left(u\left(x_{1}, y_{1}\right), \ldots, u\left(x_{n}, y_{n}\right)\right) \quad$ is strictly quasi-concave in $\mathbb{R}_{++}^{2 n}$, and such that $W\left(u\left(x_{1}, y_{1}\right), \ldots, u\left(x_{n}, y_{n}\right)\right)>W(u(0), \ldots, u(0))$ implies $u\left(x_{i}, y_{i}\right)>u(0)$ for all $i$

The utilitarian sum of hospitals' utility functions $\sum_{i \in N} u:(x, y) \rightarrow \sum_{i \in N} u\left(x_{i}, y_{i}\right)$ yields an example of a social utility function that verifies Assumption 3. Assumption 3 is maintained throughout the sequel. We can now introduce, as formal Definitions 3 and 4 below, two derived notions that will prove useful for the normative appreciation of production equilibrium, namely, the socially efficient production of final care services of the medical care system (in short, social optimum), and the social scarcity of (potential) graft resources.

Definition 3: A final production combination $(x, y)$ of the medical care system, or associate inputoutput combination $(x, y, z, v, t)$, is socially efficient if it maximizes the social utility function $W$ in the set of socially accessible input-output combinations $\left\{(x, y, z, v, t) \in \mathbb{R}_{+}^{7 n}: \sum_{i \in N} t_{i} \leq \sum_{i \in N} z_{i} ; \quad z \leq \omega ; \sum_{i \in N} v_{i}^{x}+v_{i}^{y} \leq n B ;\right.$ and $\left(x_{i}, y_{i}, z_{i}\right) \leq g\left(t_{i}, v_{i}\right)$ for all $\left.\mathrm{i}\right\} .{ }^{10}$

Definition 4: The potential graft resources of hospital $i$ are socially scarce if a ceteris paribus increase in this hospital's endowment increases optimal social utility, that is, if $\max \left\{W\left(u\left(x_{1}, y_{1}\right), \ldots, u\left(x_{n}, y_{n}\right)\right):(x, y, z, v, t) \geq 0 ; \sum_{i \in N} t_{i} \leq \sum_{i \in N} z_{i} ; z \leq \omega^{\prime} ; \sum_{i \in N} v_{i}^{x}+v_{i}^{y} \leq n B ;\right.$ and $\left(x_{i}, y_{i}, z_{i}\right)$ $\left.\leq g\left(t_{i}, v_{i}\right) \forall i\right\}>\max \left\{W\left(u\left(x_{1}, y_{1}\right), \ldots, u\left(x_{n}, y_{n}\right)\right):(x, y, z, v, t) \geq 0 ; \sum_{i \in N} t_{i} \leq \sum_{i \in N} z_{i} ; z \leq \omega^{\prime} ; \sum_{i \in N} v_{i}^{x}+v_{i}^{y} \leq\right.$ $n B$; and $\left.\left(x_{i}, y_{i}, z_{i}\right) \leq g\left(t_{i}, v_{i}\right) \forall i\right\}$ whenever $\omega_{i}^{\prime}>\omega_{i}$ and $\omega_{n / i}^{\prime}=\omega_{n / i}$.

The next proposition and corollary characterize social optimum and scarcity.

\footnotetext{
${ }^{10}$ Note that the specification of the social opportunity set implied by this definition of the social optimum supposes, as already stated at the end of 3-2 above, that the constraints binding the production of final transplant care services, if any, are the rationing constraints over graft production, as opposed to the budget constraints limiting purchases of general inputs.
} 
Theorem 2: There exist social optima $\left(x^{*}, y^{*}, z^{*}, v^{*}, t^{*}\right)$, which are $\gg 0$, with a unique optimal production of final care services $\left(x^{*}, y^{*}\right)$, and verify the following system of necessary and sufficient first-order conditions, where partial derivatives are evaluated at the optimum: (i) $\sum_{i \in N} t_{i}^{*}=\sum_{i \in N} z_{i}^{*}$; (ii) $\sum_{i \in N} v_{i}^{x^{*}}+v_{i}^{y^{*}}=n B$; (iii) for all $i,\left(x_{i}^{*}, y_{i}^{*}, z_{i}^{*}\right)=g\left(t_{i}^{*}, v_{i}^{*}\right)$ and $\frac{\partial_{1} u}{\partial_{2} u}=\frac{\partial_{2} g^{y}}{\partial_{2} g^{x}}$; (iv) $z^{*} \leq \omega$ and there exists $(\lambda, \delta) \in \mathbb{R}_{++} \times \mathbb{R}_{+}^{n}$ such that $\partial_{i} W . \partial_{1} u . \partial_{1} g^{x}=\lambda, \delta_{i}=\lambda+\frac{\partial_{i} W}{\partial_{2} g^{z}+\partial_{3} g^{z}} .\left(\partial_{1} u . \partial_{3} g^{x}+\partial_{2} u . \partial_{3} g^{y}\right)<\lambda$, and $\delta_{i} \cdot\left(\omega_{i}-z_{i}^{*}\right)=0$ for all $i$, where $\lambda$ and $\delta_{i}$ are the marginal social utilities of aggregate hospitals' contribution and hospital $i$ 's potential graft resources respectively. The potential graft resources of hospital $i$ are scarce at social optimum if and only if $\delta_{i}$ is $>0$.

Corollary 1: $\left(x^{*}, y^{*}, z^{*}, t^{*}\right)$ is socially optimal if and only if: (i) $\sum_{i \in N} t_{i}^{*}=\sum_{i \in N} z_{i}^{*}$; (ii) for all $i$, $y_{i}^{*}=F\left(x_{i}^{*}, z_{i}^{*}, t_{i}^{*}\right)$ and $\frac{\partial_{1} u\left(x_{i}^{*}, y_{i}^{*}\right)}{\partial_{2} u\left(x_{i}^{*}, y_{i}^{*}\right)}=\partial_{1} F\left(x_{i}^{*}, z_{i}^{*}, t_{i}^{*}\right) ;$ (iv) $z^{*} \leq \omega$ and there exists $(\lambda, \delta) \in \mathbb{R}_{++} \times \mathbb{R}_{+}^{n}$ such that, for all $i, \partial_{i} W \cdot \partial_{2} u . \partial_{3} F=\lambda, \delta_{i}=\partial_{i} W \cdot \partial_{2} u \cdot \partial_{3} F \cdot\left(1+\frac{\partial_{2} F}{\partial_{3} F}\right)<\lambda$, and $\delta_{i} \cdot\left(\omega_{i}-z_{i}^{*}\right)=0$, where the partial derivatives are evaluated at the optimum. Hospital $i$ 's potential graft resources are scarce at social optimum if and only if this hospital's MRC is $<1$ at the optimum.

Corollary 1 does not require an explicit proof, as simple consequence of Theorem 2 and Lemma 1. The proof of Theorem 2 is detailed in the appendix (A-2).

To sum up, in a context of scarcity of transplant and general inputs of the health system, and in the absence of a market for the former, socially optimal provision of transplant care services should be limited by hospital's rationing constraints $z_{i} \leq \omega_{i}$, implying $z=\omega$ at social optimum, while autarkic provision should be limited by hospitals' autarkic budget constraints $v_{i}^{x}+v_{i}^{y}+v_{i}^{z} \leq B$, at least for those hospitals that are best endowed in terms of their potential graft resources, implying the waste of a fraction of the latter, that is, a socially inefficient (under-) provision of transplants (both intermediary 
and final). This yields the basic rationale, within our abstract representation of the medical care system, for the introduction of a transplant agency in charge of collecting and circulating grafts in the place of the missing market for transplant inputs.

\section{4-2-Regulated equilibrium with public good interactions}

We suppose, from there on, that there exists a transplant agency of the type described at the beginning of this section, and moreover assume that this agency endorses the social preferences of Assumption 3 . As noted above, the existence of a transplant agency induces public good externalities of the technological type between hospitals, as long as the latter control their production of grafts, that is: The graft production decided by any hospital has consequences on the production sets of all others through transplant redistribution by the agency.

Public good interactions between hospitals and the agency are modelled below through a device which has become standard in mechanism design theory, namely, subgame-perfect Nash equilibria of twostage games (see notably, in the context of models of private contributions to a public good, Guttman (1978, 1987), Bergstrom (1989) or Cornes and Silva (1999) and also the detailed references reviewed in Mercier Ythier (2006: 6.3 and A.2.1)). We successively consider three possible variants of the twostage game, where hospitals and the agency alternate as first and second players in the game. The first two are defined below as the myopic game (4-2-1) and the clear-sighted game (4-2-2) respectively. The third notion of two-stage game, labelled monitored game, is defined and studied in subsection 4-3.

\section{4-2-1-Myopic equilibrium}

In the first variant of the two-stage game, hospitals play second, each one solving $\max \left\{u\left(x_{i}, y_{i}\right):\left(x_{i}, y_{i}, z_{i}, v_{i}\right) \in A_{i}\left(t_{i}, \omega_{i}\right)\right\}$ with respect to $\left(x_{i}, y_{i}, z_{i}, v_{i}\right)$ for any given $t_{i}$. We denote by $\varphi_{i}$ hospital $i$ 's reaction correspondence at this stage, defined by $\varphi_{i}(t)=\arg \max \left\{u\left(x_{i}, y_{i}\right):\left(x_{i}, y_{i}, z_{i}, v_{i}\right) \in A_{i}\left(t_{i}, \omega_{i}\right)\right\}$, and let $\varphi=\left(\varphi_{1}, \ldots, \varphi_{n}\right)$. The transplant agency plays 
in the first stage of the game, solving $\max \left\{W\left(u\left(x_{1}, y_{1}\right), \ldots, u\left(x_{n}, y_{n}\right)\right):(x, y, z, v) \in \varphi(t)\right.$ and $\left.t \in A_{0}(z)\right\}$

with respect to $t$. An equilibrium of the game is a state $(x, y, z, v, t)$ that solves the latter program. We refer to this first notion of equilibrium as the myopic equilibrium in the sequel, due to the short-sighted free-riding behaviour of hospitals which it implies.

We have the following simple benchmark property for the myopic equilibrium:

Theorem 3: The provision of transplant care services and grafts is null at myopic equilibrium.

Proof: Graft production is costly for the hospital, due to its crowding effects on hospital's production of final care services $\left(x_{i}, y_{i}\right)$, and doesn't yield any advantage, ceteris paribus (that is, given others' graft provision and agency's transfers), in terms of hospital's utility. Therefore $z=0$, which implies $t=0$, which implies in turn $x=0 . \mathbf{m}^{11}$

The above result is interesting as a clear-cut, albeit extreme expression of the coordination problem of transplant activities. It is individually rational for myopic hospitals to free ride, or shirk, on graft production, that is, to attempt to shift over the others the crowding costs induced by graft production ${ }^{12}$. Myopia interprets as a lack of understanding, at individual level, of the collective damages that result from generalized free-riding, namely, the dramatic under-provision of transplants (no provision at all, in the case under consideration). The existence of a central agency collecting and redistributing transplants is not only insufficient, per se, for solving the public good problem; it dramatically

11 The set of myopic equilibria is $\left\{(x, y, z, v, t) \in \mathbb{R}_{+}^{7 n}: x=z=t=0 ; v_{i}^{z}=0, v_{i}^{x}+v_{i}^{y} \leq B / w\right.$ and $0 \leq y_{i} \leq$ $g^{y}\left(0, v_{i}^{y}, v_{i}^{x}+v_{i}^{y}\right)$ for all $\left.i\right\}$, implying an equilibrium utility of hospitals and the agency everywhere equal to their minimal values in their respective domains, that is $u(0)$ and $W(u(0), \ldots, u(0))$ respectively.

${ }^{12}$ Note that the formulation of the public good problem as a pure coordination problem here and below does not rely on imperfect or costly information. The reason for this is empirical: accounts of the Spanish and other experiences of national transplant systems we are aware of put little emphasis, if any emphasis at all, on information problems per se. The main difficulty, as far as production units are concerned, seems to be selfcentredness, understood as the propensity of each hospital to concentrate on its own patients, and subsequent reluctance to consider costly actions that are not directly related to this priority. One of the main lessons of the Spanish experience, it seems to us, is that most problems are solved by simply discharging hospitals, in some appropriate way, of the concern of on site organization of graft production (including identification of potential donors, and donations interviews). 
deteriorates production equilibrium, relative to the autarkic equilibrium, if public good interactions are of the myopic type.

\section{4-2-2-Clear-sighted equilibrium}

The consequence of Theorem 3 is too extreme to be accepted literally. Hospitals should be, and actually are well aware of the damages of shirking (in the sense of footnote ${ }^{11}$ ) for the medical care system as a whole, and for themselves as a part of it. Myopia does not appear a realistic assumption, in other words, both a priori and in view of its logical implication.

In the variant of the two-stage game that we introduce now, the agency plays second, solving $\max \left\{W\left(u\left(x_{1}, y_{1}\right), \ldots, u\left(x_{n}, y_{n}\right)\right):\left(x_{i}, y_{i}, z_{i}, v_{i}\right) \in A_{i}\left(t_{i}, \omega_{i}\right)\right.$ for all $i$, and $\left.t \in A_{0}(z)\right\} \quad$ with $\quad$ respect to $(x, y, v, t)$ for any given $z \leq \omega$. We denote by $\varphi^{0}=\left(\varphi_{1}^{0}, \ldots, \varphi_{n}^{0}\right)$ the agency's transfer correspondence at this stage, where $\varphi_{i}^{0}$ yields the agency's optimal transfers to hospital $i$ for any fixed $z$. Hospitals play first, each one solving $\max \left\{u\left(x_{i}, y_{i}\right):\left(x_{i}, y_{i}, z_{i}, v_{i}\right) \in A_{i}\left(t_{i}, \omega_{i}\right) ; t_{i} \in \varphi_{i}^{0}(z)\right\}$ with respect to $\left(x_{i}, y_{i}, z_{i}, v_{i}\right)$ for any given vector of graft production of other hospitals $z_{n / i}$. An equilibrium of the game is a Nash non-cooperative equilibrium of the first-stage game, that is, a state $\left(x^{*}, y^{*}, z^{*}, v^{*}, t^{*}\right)$

such that: $t^{*} \in \varphi^{0}\left(z^{*}\right) ; \quad$ and for all $i, \quad\left(x_{i}^{*}, y_{i}^{*}, z_{i}^{*}, v_{i}^{*}\right) \quad$ solves $\max \left\{u\left(x_{i}, y_{i}\right):\left(x_{i}, y_{i}, z_{i}, v_{i}\right) \in A_{i}\left(t_{i}, \omega_{i}\right) ; t_{i} \in \varphi_{i}^{0}\left(\left(z_{n / i}^{*}, z_{i}\right)\right)\right\}$. We name it the clear-sighted equilibrium in the sequel, because it embodies hospitals' clear awareness of the public good externality associated with graft production, and individual damages from free-riding behaviour that it implies for them.

We establish below that clear-sightedness, if it actually improves the functioning of the transplant care system relative to the myopic game, by implying a positive production of grafts and transplant services (Theorem 4-(i)), nevertheless does not suffice for solving the under-provision problem. Precisely, it is shown that a fraction of the system's resources for graft production remains unexploited, in general, at clear-sighted equilibrium when graft resources are socially scarce (Theorem 4-(ii)). 
We restrict attention, in this subsection, to the medical care systems that have clear-sighted equilibria. The existence property of clear-sighted equilibrium is analyzed in detail in the appendix (A-4). It is shown there (Lemma 5) that the critical feature which conditions existence is that hospitals' first-stage reaction correspondences be convex-valued. A minimal sufficient condition on preferences and technology for the latter is that the first-stage reduced form of hospitals' utility functions $\left(x_{i}, z_{i}\right) \rightarrow u\left(x_{i}, F\left(x_{i}, z_{i}, \varphi_{i}(z)\right)\right.$ be quasi-concave (A-4: Lemma 4). Formally:

Definition 5: The medical care system $(W, u, g, \omega)$ is convex if, for all $i$, the first-stage reduced form of hospital $i$ 's utility function $\left(x_{i}, z_{i}\right) \rightarrow u\left(x_{i}, F\left(x_{i}, z_{i}, \varphi_{i}\left(\left(z_{n / i}^{*}, z_{i}\right)\right)\right)\right.$ is quasi-concave over $\left\{\left(x_{i}, z_{i}\right) \in \mathbb{R}_{+}^{2}: 0<z_{i} \leq \omega_{i}\right\} \quad$ for $\quad$ all $\quad z^{*} \in\left\{z \in \mathbb{R}_{+}^{n}: z \leq \omega\right\} \quad$ and quasi-concave over $\left\{\left(x_{i}, z_{i}\right) \in \mathbb{R}_{+}^{2}: 0 \leq z_{i} \leq \omega_{i}\right\}$ for all $z^{*} \in\left\{z \in \mathbb{R}_{+}^{n}: z \leq \omega ; 0<z_{n / i}\right\}$.

Theorem 4: Let $(W, u, g, \omega)$ be convex. (i) Clear-sighted equilibria exist and are $\gg 0$. (ii) Transplant care services are underprovided, in general, at clear-sighted equilibrium (that is, equilibrium graft production is $<\omega$ ) when hospitals' graft resources are all scarce at social optimum. (iii) Clear-sighted equilibrium is a social optimum notably if programs $\max \left\{u\left(x_{i}, F\left(x_{i}, z_{i}, z_{i}\right)\right): z_{i} \leq \omega_{i}\right\}$ yield a same solution $\left(x_{i}, z_{i}\right)$ for all hospitals.

The details of the proof are given in the appendix (A-5). We concentrate here on the essence of the argument underlying the second and third parts of the theorem, beginning with the optimality property of the latter.

The first-order conditions characterizing social optimum (Corollary 1) and clear-sighted equilibrium (Lemmas 2 and 3) differ on a single essential point, namely, hospitals' marginal utilities of graft resources, which read $\partial_{2} u \cdot \partial_{3} F \cdot\left(1+\frac{\partial_{2} F}{\partial_{3} F}\right)\left(=\delta_{i} / \partial_{i} W\right)$ for social optimum and $\partial_{2} u \cdot \partial_{3} F \cdot\left(\partial_{i} \varphi_{i}^{0}+\frac{\partial_{2} F}{\partial_{3} F}\right)$ 
for social equilibrium ${ }^{13}$. The transfer policy $\varphi^{0}$ of the transplant agency will therefore completely solve the coordination problem of the care system, that is, make hospitals' equilibrium and optimum evaluation of graft resources coincide in all circumstances if, and in general only if $\partial_{i} \varphi_{i}^{0}(z)=1$ for all $i$ and all $z$, that is, if $\varphi^{0}$ is the identity function $z \rightarrow z$ of $\mathbb{R}^{n}$. The latter transfer policy consists of returning each hospital its contribution in all circumstances.

Clearly enough, this perfect or complete solution to the coordination problem should, in general, conflict with the end-objectives of allocation efficiency and distribution equity implied by the social preference relation. In other words, returning each hospital its contribution in all circumstances is generally not an optimal transfer policy of the transplant agency at the second stage of the clearsighted game. The main first-order conditions for the latter are $\partial_{i} W . \partial_{2} u . \partial_{3} F=\lambda$, equating marginal social utilities of transfers to the marginal social utility of hospitals' aggregate contribution ${ }^{14}$. The anonymity and convexity properties of the social preference relation (Assumption 3) imply that marginal social utilities $\partial_{i} W$ are identical if and only if hospitals' utilities are identical (and $>u(0)$ ). The identity function $z \rightarrow z$ solves, therefore, the agency's second-stage problem if, and in general only if the following two conditions hold: (i) a constant function $z_{i} \rightarrow \partial_{3} F\left(x_{i}, z_{i}, z_{i}\right)$; (ii) and hospital's first-stage reduced form programs $\max \left\{u\left(x_{i}, F\left(x_{i}, z_{i}, z_{i}\right)\right): z_{i} \leq \omega_{i}\right\}$ yielding a same solution $\left(x_{i}, z_{i}\right)$ for all hospitals.

Condition (ii) above interprets as follows: Hospitals, producing their transplant care services from their own production of transplant inputs, and financing their purchases of general inputs for their intermediary graft production from the agency's budget, choose the same production combination $\left(x_{i}, F\left(x_{i}, z_{i}, z_{i}\right), z_{i}\right)=\left(x^{*}, y^{*}, z^{*}\right)$ in their individual opportunity sets. In terms of the rationing

\footnotetext{
${ }^{13}$ The other difference lies in the specification of budget constraints, namely, aggregate budget constraint at social optimum versus individual budget constraints at equilibrium. We assume implicitly here and explicitly in the case of the monitored equilibrium studied in the next subsection that social optimum is always decentralizable, in the sense that if an input-output combination of the care system can be achieved from its aggregate budget, then it can also be achieved from the set of hospitals' individual budgets. This assumption is not much demanding in our setup, since hospitals are assumed identical in all respects except potential graft endowment and social optimum verifies hospitals' rationing constraints by definition.

${ }^{14}$ The other conditions that are relevant for allocation efficiency are the first-order conditions equating hospitals' marginal rates of substitution with their marginal rates of transformation. This set of conditions is automatically verified, in a decentralized way, at the solutions of hospitals' first-stage programs of the clear-sighted game.
} 
constraints over graft production, this type of configuration is compatible with only two possibilities: either none of these constraints are binding; or all hospitals' endowments are equal, if a constraint is binding for some hospital (that is, satiated, with positive associate hospitals' marginal utility of endowment). We establish in the appendix the intuitively clear fact that, in such cases, status quo transfers $\varphi^{0}\left(z^{*} \cdot e_{n}\right)=z^{*} \cdot e_{n}$ are equilibrium transfers, and that $\left(x^{*} \cdot e_{n}, y^{*} \cdot e_{n}, z^{*} \cdot e_{n}\right)$ is a socially efficient clear-sighted equilibrium production combination for such transfers. Example 2 below yields an instance of a calculable care system with constant unitary MRC where the optimality property of Theorem 4-(iii) applies and status quo is the agency's transfer policy.

To sum up, the agency's end-objectives of allocation efficiency and distribution equity should conflict with coordination objectives in most circumstances, the only notable exception corresponding to the case where hospitals spontaneously achieve social optimum because rationing constraints are either non-binding or identical for all of them. In realistic circumstances, where rationing constraints are binding at social optimum and there is some diversity in hospitals' endowments, the status quo transfer policy which consists of returning each hospital its contribution cannot be an optimal policy (that is, cannot yield equal social marginal utilities of transfers for all hospitals), at social optimum and in the close neighbourhood, for anonymous, increasing, strictly convex social preferences. The agency's transfer policy is therefore bound to introduce some discrepancies between hospitals' marginal evaluations of their graft resources computed from the standpoint of social optimum $\left(\partial_{2} u . \partial_{3} F \cdot\left(1+\frac{\partial_{2} F}{\partial_{3} F}\right)\right)$ and equilibrium $\left(\partial_{2} u . \partial_{3} F \cdot\left(\partial_{i} \varphi_{i}^{0}+\frac{\partial_{2} F}{\partial_{3} F}\right)\right)$, through hospitals' marginal returns of individual contribution $\partial_{i} \varphi_{i}^{0}$ distinct from 1 .

In the practically relevant case where graft resources are scarce at social optimum (that is, marginal rates of compensation $-\frac{\partial_{2} F}{\partial_{3} F}<1$ for all $i$ at social optimum, implying that socially optimal graft production is $z=\omega$ ) social optimum is not a clear-sighted equilibrium if and only if the marginal return of contribution of some hospital is smaller than its marginal rate of compensation, that is, if and only if $\partial_{i} \varphi_{i}^{0}(\omega)<-\frac{\partial_{2} F}{\partial_{3} F}$ for some $i$, implying that hospital $i$ then wants to deviate from socially 
optimal $z_{i}=\omega_{i}$ by diminishing its contribution. In Example 1 below, we present a family of calculable care systems, with symmetric log-linear utility function of hospitals, additive utilitarian social utility function, and square root Cobb-Douglas production functions, where the agency's second-stage optimal transfer policy turns out to be the equal sharing of aggregate contribution, that is, $\varphi_{i}^{0}(z)=\frac{1}{n} \sum_{i \in N} z_{i}$ for all $i$ for all $z$. Hospitals' marginal return of own contribution $\partial \varphi_{i}^{0}(z)$ therefore is equal to $1 / n$ in the example, hence smaller than 1 if there is more than one hospital and decreasing to 0 as hospitals' number grows to infinity. We show that the Nash equilibrium of the first stage of the clear-sighted game, which coincides by definition with clear-sighted equilibrium for agency's optimal policy above, reduces to an example of the general class of symmetric Nash equilibrium with public goods of Chamberlin $(1974)^{15}$ when hospitals' number is sufficiently large. In particular: hospitals' individual contribution is positive, decreasing in hospitals' number, and tends asymptotically to 0 as the latter grows to infinity; hospitals' aggregate contribution increases in hospitals' number, at a lower speed than the latter. This notably implies that no rationing constraint is binding in first-stage hospitals' programs, and that clear-sighted equilibrium is therefore independent of the initial distribution of potential graft resources, when the number of hospitals is sufficiently large. Moreover, we show that graft resources are scarce at social optimum, whatever the number of hospitals, for suitable equal distributions of potential graft resources. Transfers actually practiced by transplant agencies certainly are much closer to equal sharing policy $z \rightarrow \frac{1}{n} \sum_{i \in N} z_{i}$ than to status quo policy $z \rightarrow z$, so that hospitals' marginal returns of contribution $\partial \varphi_{i}^{0}(z)$ should be considered much closer to $1 / n$ than to 1 in reality, hence much closer to 0 than to 1 in view of actual numbers of care production units in charge of providing the transplant care services (155 in the case of Spain, for example: see section 2 above). Inefficient under-provision therefore seems a more plausible outcome of the clear-sighted game, for realistic assumptions on preferences, technology and number of production units.

\footnotetext{
${ }^{15}$ See also the generalizations and extensions of Chamberlin's result by Andreoni (1988) and Fries et alii (1991), and the related literature reviewed in Mercier Ythier (2006: 6.2)
} 
Theorem 4-(iii) is a rotten kid theorem $(\operatorname{Becker}(1974,1981))$. Precisely, it identifies configurations of principal-agent interactions where the optimal transfer policy of the (benevolent) principal drives the (non-cooperative, self-centred) agents to implement a social optimum which coincides with the principal's optimum. The conditions under which this property obtains in this model of the transplant care system are quite different from Becker's $(1974,1981)$, Bergstrom's (1989) and also from Cornes and Silva's (1999).

The public good externalities of first-stage equilibrium pre-exist to transfer policy in the game of Cornes and Silva. This and the neutrality property of transfers allow the principal to use transfer policy as a pure coordination device in their setup: in the absence of any trade off between allocation and distribution objectives (due to neutrality), the principal's optimal transfer policy achieves social optimum by equating individual marginal valuations of the public and private goods at first-stage equilibrium with their marginal valuations at social optimum. The public good externalities of the first-stage equilibrium of the transplant care game, if any, are, by contrast, generated by the principal's transfer policy (as in Becker's and Bergstrom's game); moreover, transfer policy induces public good externalities if and only if it is not of the status quo type, that is, if and only if it does not merely consist of returning each agent its contribution. Only if status quo is the agency's optimal transfer policy can the mechanism of Cornes and Silva be successfully replicated in the context of the transplant care game, that is, use transfer policy as a pure coordination device for achieving social optimum. Theorem 4-(iii) gives the sufficient, and in general necessary, condition for status quo transfer policy to be the agency's second-stage optimal policy.

Bergstrom (1989) states that the rotten kid theorem applies if, and in general only if, agents' utilities are conditionally transferable. Bergstrom's general property, like Becker's original theorem, do not apply to the transplant care game if rationing constraints are binding at social optimum. Becker's theorem applies in the context of competitive market exchange, essentially because (perfect) competitive exchange automatically achieves allocation efficiency for any distribution of money income, thereby allowing the principal to optimize the sole distribution of income, by means of lumpsum endowment (or numeraire) transfers (see the Hick's composite theorem in Bergstrom (1989), and also the Example 2 of Mercier Ythier (2007)). The rotten kid property still obtains outside competitive 
market exchange if the allocation efficiency frontier is invariant to redistribution and if the principal can freely redistribute aggregate money income between self-centred agents (Bergstrom (1989): Proposition 1). None of these conditions apply to the transplant care game, except in the special case where rationing constraints are non-binding at equilibrium (see the argument of footnote ${ }^{16}$ and the proof of Theorem 4-(iii) in the appendix). Moreover, the ban on markets of transplant inputs, which makes competitive markets fail in the transplant economy, also is the main explanation, both in theory and in practice, for the existence of binding rationing constraints in the production of transplant care services. The virtuous rotten kids of Becker's theorem are, so to speak, daughters and sons of abundance.

\section{Example 1: A calculated example of Olson-Chamberlin underprovision}

We study the following calculable medical care system $(W, u, g, \omega)$ : production functions are the concave Cobb-Douglas of $g\left(t_{i}, v_{i}\right)=\left(v_{i}^{x}+v_{i}^{y}+v_{i}^{z}\right)^{-\frac{1}{2}}\left(\left(t_{i} v_{i}^{x}\right)^{\frac{1}{2}},\left(v_{i}^{y}\right)^{\frac{1}{2}},\left(v_{i}^{z}\right)^{\frac{1}{2}}\right)$; hospitals' utility function is the $\log$ linear $u\left(x_{i}, y_{i}\right)=\log x_{i}+\log y_{i} ; \quad$ social utility function is the utilitarian sum

\footnotetext{
${ }^{16}$ Transferable utility translates as follows into our framework: The preference relation underlying hospitals' (identical) reduced form utility functions $u\left(x_{i}, F\left(x_{i}, z_{i}, t_{i}\right)\right)$ admits a utility representation of the type A. $t_{i}+C\left(x_{i}, z_{i}\right)$, where $A$ is a $>0$ real number and $C$ is a real-valued function decreasing in $z_{i}$. Suppose for simplicity (without significant loss of generality by Mas-Colell (1985): 2.3.11) that $u\left(x_{i}, F\left(x_{i}, z_{i}, t_{i}\right)\right)=A . t_{i}+C\left(x_{i}, z_{i}\right)$ for all $i$. This implies $\partial_{2} F / \partial_{3} F=\left(\partial_{2} u . \partial_{2} F\right) /\left(\partial_{2} u . \partial_{3} F\right)=\partial_{2} C / A$, where the points of evaluation of partial derivatives are omitted to alleviate notations. Denoting by $u_{i}$ a utility level of hospital $i$, the utility possibility set conditional on the system of agents' actions $(x, z)$ is the simplex $\left\{\left(u_{1}, \ldots, u_{n}\right) \geq\left(C\left(x_{1}, z_{1}\right), \ldots, C\left(x_{n}, z_{n}\right)\right): \sum_{i \in N} u_{i} \leq A \cdot \sum_{i \in N} z_{i}+\sum_{i \in N} C\left(x_{i}, z_{i}\right)\right\}$. The rotten kid theorem implies the maximization of "social income" $A \cdot \sum_{i \in N} z_{i}+\sum_{i \in N} C\left(x_{i}, z_{i}\right)$ relative to socially accessible agents' actions $(x, z)$ at equilibrium. The f.o.c. for a maximum of $A \cdot \sum_{i \in N} z_{i}+\sum_{i \in N} C\left(x_{i}, z_{i}\right)$ such that $x \gg 0$ subject to rationing constraints $z \leq \omega$ read: $\partial_{1} C\left(x_{i}, z_{i}\right)=0,1+\frac{\partial_{2} C}{A} \geq 0$ and $\left(1+\frac{\partial_{2} C}{A}\right) \cdot\left(\omega_{i}-z_{i}\right)=0$ for all $i$, and therefore coincide with the f.o.c. for the solutions of $\max \left\{u\left(x_{i}, F\left(x_{i}, z_{i}, z_{i}\right)\right): z_{i} \leq \omega_{i}\right\}$ with positive $x_{i}$. Supposing an anonymous utility function of the principal, this set of conditions characterize a socially optimal clear-sighted equilibrium, with status quo second-stage optimal transfer policy $\varphi^{0}: z \rightarrow z$, if and only if programs $\max \left\{u\left(x_{i}, F\left(x_{i}, z_{i}, z_{i}\right)\right): z_{i} \leq \omega_{i}\right\}$ have a same solution, that is, if and only if rationing constraints are either nonbinding in all these programs or identical in all of them (the latter implying identical hospitals' endowments). In particular, Proposition 1 of Bergstrom (1989) does not apply if, and in general only if, distinct rationing constraints are binding in at least two of these programs. The assumption of Bergstrom's proposition that fails to hold, in general, in the latter case is that the principal can choose any vector of transfers in set $\left\{t \in \mathbb{R}_{+}^{n}: \sum_{i \in N} t_{i} \leq \sum_{i \in N} z_{i}\right\}$, implying that the principal's transfers are not limited by rationing constraints in the cases covered by the proposition.
} 
$W\left(u_{1}\left(x_{1}, y_{1}\right), \ldots, u_{n}\left(x_{n}, y_{n}\right)\right)=\sum_{i \in N} u\left(x_{i}, y_{i}\right)$. It verifies assumptions 1, 2 and 3. Associate function $F$ reads $F\left(x_{i}, z_{i}, t_{i}\right)=\left(1-z_{i}^{2}-\frac{x_{i}^{2}}{t_{i}}\right)^{\frac{1}{2}}$. The first-order conditions of Lemma 2 then yield $\varphi_{i}^{0}(z)=(1 / n) \sum_{i \in N} z_{i}$ for all $i$, that is, the agency's optimal distribution policy is equal sharing of aggregate hospitals' contribution. ${ }^{17}$ Substituting optimal transfer $\varphi_{i}^{0}(z)$ for $t_{i}$ in $F$ yields the following reduced form for hospital $i$ 's first stage objective function: $u\left(x_{i}, F\left(x_{i}, z_{i}, \varphi_{i}^{0}(z)\right)\right)=\log x_{i}+(1 / 2) \log \left(1-z_{i}^{2}-n \frac{x_{i}^{2}}{\sum_{j \in N} z_{j}}\right)$, viewed as a function of $\left(x_{i}, z_{i}\right)$ for fixed $z_{n / i}$. The path of hospital $i$ 's optimal final production conditional on $z_{i}$ is $\left.\left\{\left(t_{i} \frac{1-z_{i}^{2}}{2}\right)^{\frac{1}{2}},\left(\frac{1-z_{i}^{2}}{2}\right)^{\frac{1}{2}}\right): 0 \leq z \leq \omega_{i}\right\}$. By further restricting to this path the objective function above, we get the following final reduced form for hospital $i$ 's first stage program $\max \left\{(1 / 2) \log \sum_{j \in N} z_{j}+\log \left(1-z_{i}^{2}\right)-(1 / 2) \log n-\log 2: 0 \leq z_{i} \leq \omega_{i}\right\}$, where the objective function is (differentiably) strictly concave. Let us provisionally ignore the rationing constraint in the latter program. The first-order necessary and sufficient condition for an unconstrained maximum reads $5 z_{i}^{2}+4\left(\sum_{j \in N: j \neq i} z_{j}\right) z_{i}-1=0 . \quad$ Solving for $z_{i}$ yields the unique $>0$ solution $z_{i}=-(2 / 5) \sum_{j \in N: j \neq i} z_{j}+(1 / 5) \sqrt{5+4\left(\sum_{j \in N: j \neq i} z_{j}\right)^{2}}$. Letting $z_{i}=z^{*}$ for all $i$ in the solution and solving for $z^{*}$ yields the symmetric individual contribution $z^{*}=\frac{1}{\sqrt{4 n+1}}$. In particular, there exists $n_{0}$ such that $z^{*} \cdot e_{n} \ll \omega$ for all $n \geq n_{0}$, implying that $z^{*}$ is a symmetric equilibrium contribution of the medical care system, with non-binding rationing constraints, when the number of hospitals is at least as large as $n_{0}$. This is then the unique equilibrium contribution, as a special case of Cornes and Hartley,

\footnotetext{
17 Notably : f.o.c. $\frac{\partial_{1} u\left(x_{i}, F\left(x_{i}, z_{i}, t_{i}\right)\right)}{\partial_{2} u\left(x_{i}, F\left(x_{i}, z_{i}, t_{i}\right)\right)}=-\partial_{1} F\left(x_{i}, z_{i}, t_{i}\right)$ yields $\frac{x_{i}^{2}}{t_{i}}=y_{i}{ }^{2}$ for all $i$; substituting into f.o.c. $\partial_{2} u\left(x_{i}, F\left(x_{i}, z_{i}, t_{i}\right)\right) . \partial_{3} F\left(x_{i}, z_{i}, t_{i}\right)=\lambda$ and adding up over $i$ then yields both $\lambda=2 n / \sum_{j \in N} z_{j}$ and $t_{i}=(1 / n) \sum_{j \in N} z_{j}$.
} 
2007. ${ }^{18}$ Equilibrium individual contribution lies in $] 0,1\left[\right.$ for all $n \geq n_{0}$. It is decreasing, asymptotically equivalent to $\frac{1}{2 \sqrt{n}}$, converging to 0 as the number of hospitals grows to infinity, while aggregate equilibrium contribution $\sum_{i \in N} z_{i}=\frac{n}{\sqrt{4 n+1}}$ is increasing, growing to infinity with the number of hospitals but at a lower speed than the latter. ${ }^{19}$ One verifies easily from the first-order conditions of Theorem 2 that these equilibria are socially inefficient. The marginal social utility of hospitals' aggregate contribution is $\lambda=\frac{n}{2 \sum_{i \in N} z_{i}}$, and the marginal social utility of hospital $i$ 's potential graft resources is $\delta_{i}=\frac{n}{2 \sum_{i \in N} z_{i}}-2 \frac{z_{i}}{1-z_{i}^{2}}$ for all $i$ in the f.o.c.. Letting $z_{i}=\frac{1}{\sqrt{4 n+1}}$ for all $i$ in the latter yields positive values of $\delta_{i}$ for all $n \geq 2$, which are inconsistent with social optimality for $n \geq n_{0}$ (since $\frac{1}{\sqrt{4 n+1}}<\omega_{i}$ for all $i$ then). Suppose, finally, that initial endowments are equally distributed, that is, $\omega=\tilde{\omega} \cdot e_{n}$ for some $\tilde{\omega} \in \mathbb{R}_{++}$for all $n$. The MRC at $\left(x_{i}, z_{i}, t_{i}\right) \gg 0$ is $2 z_{i}\left(t_{i} / x_{i}\right)^{2}$. On the path of hospital $i$ 's optimal final production conditional on $z_{i}$, and for agency's optimal

\footnotetext{
${ }^{18}$ The reader can check this by proceeding to the following change of variable: Let the utility function in the framework of Cornes et alii be $U\left(x_{i}, G\right)=\log \left(-x_{i}^{2}+2 x_{i}\right)+(1 / 2) \log \sum_{j \in N} g_{j}+-(1 / 2) \log n-\log 2$, where $x_{i}$ denotes their "private good" (not to be confused with our "provision of transplant care services"), $G=\sum_{j \in N} g_{j}$ is the public good, and $g_{j}$ is $j$ 's individual contribution to $G$. Let their agent's endowment (not to be confused with our "potential of graft production") be $=1$. Their reduced utility function, obtained by substituting budget constraint $x_{i}+g_{i}=1$ in the former, is $U\left(1-g_{i}, g_{i}+G_{-i}\right)=$ $\log \left(1-g_{i}{ }^{2}\right)+(1 / 2) \log \left(g_{i}+G_{-i}\right)+-(1 / 2) \log n-\log 2$, where $G_{-i}=\sum_{j \in N: j \neq i} g_{j}$, which is identical to the reduced form of the utility function of our calculated example. A simple calculation shows that function $U\left(1-g_{i}, g_{i}+G_{-i}\right)$ verifies the normality condition of Chamberlin (1974), which implies in turn the condition for uniqueness of Cornes and Hartley (2007).

${ }_{19} \frac{n}{\sqrt{4 n+1}}$ is asymptotically equivalent to $\frac{1}{2} \sqrt{n}$. Its instantaneous growth rate is $\frac{1}{n}-\frac{1}{2 n+\frac{1}{2}}$, which is positive and $<1$ for all $n \geq 1$, decreasing with $n$, asymptotically equivalent to $\frac{1}{2 n}$, and, in particular, tending to 0 as $n$ grows to infinity. The asymptotic behaviour of hospitals' contributions reproduces the qualitative features of the general property of Chamberlin (1974). We established in a footnote above that the first-stage Nash equilibrium of this example reduces to a special case of Chamberlin's symmetric Nash equilibrium when the number of hospitals becomes large enough to make all rationing constraints slack at equilibrium.
} 
transfer associated with $z$, this yields: $4 \cdot \frac{\sum_{i \in N} z_{i}}{n} \cdot \frac{1-z_{i}^{2}}{z_{i}}$. Therefore, hospitals' potential graft resources are all scarce at social optimum if and only if $\left.4\left(1-\tilde{\omega}^{2}\right) \in\right] 0,1[$, that is, if and only if $\tilde{\omega} \in], \frac{1}{\sqrt{5}}[$

\section{Example 2: Linear transferable transplant technology}

In this example, we consider the case of convex medical care systems with constant unitary MRC. We label this special case the transferable transplant case, by analogy with transferable utility (Bergstrom and Cornes (1983), Bergstrom and Varian (1985ab) and Bergstrom (1989)). ${ }^{20}$ We further restrict attention, for calculation purposes, to linear hospital technology. Linear technology being inconsistent with the boundary conditions of Assumption 1, we suppose, more precisely, that there exists a positive real number $\varepsilon \leq \inf \left\{\omega_{i}: i \in N\right\}$, which may be taken arbitrarily close to 0 , such that $F\left(x_{i}, z_{i}, t_{i}\right)=-a x_{i}-b z_{i}+b t_{i}+c, \quad(a, b, c) \in \mathbb{R}_{++}^{3}, \quad$ whenever $\quad\left(x_{i}, z_{i}, t_{i}, F\left(x_{i}, z_{i}, t_{i}\right)\right) \geq(\varepsilon, \mathcal{\varepsilon}, \mathcal{\varepsilon}, \mathcal{\varepsilon}) \quad($ see Figures $2 \mathrm{a}$ and $2 \mathrm{~b})$.

Let $\left(x_{i}^{*}, y_{i}^{*}\right)$ denote a local maximum of $u$ in $\left\{\left(x_{i}, y_{i}\right) \in \mathbb{R}_{+}^{2}: y_{i} \leq-a x_{i}+c\right\}$. Note that such a point: necessarily exists by continuity of $u$; is $\gg 0$ by the boundary condition of Assumption 2, and therefore is the unique global maximum of $u$ in $\left\{\left(x_{i}, y_{i}\right) \in \mathbb{R}_{+}^{2}: y_{i} \leq-a x_{i}+c\right\}$ by the strict quasiconcavity of utility in $\mathbb{R}_{++}^{2}$; is such that $y_{i}^{*}=-a x_{i}^{*}+c\left(u\right.$ being strictly increasing in $\left.\mathbb{R}_{++}^{2}\right)$; and verifies first-order condition $\frac{\partial_{1} u\left(x_{i}^{*}, y_{i}^{*}\right)}{\partial_{2} u\left(x_{i}^{*}, y_{i}^{*}\right)}=a$. We suppose in the sequel that $\left(x_{i}^{*}, y_{i}^{*}\right) \gg(\varepsilon, \varepsilon)$ (see Figure 2c).

\footnotetext{
${ }^{20}$ These characteristics of $F$ obtain easily from Assumption 1 by letting functions $g^{r}, r \in\{x, y, z\}$, be linear whenever $\left(x_{i}, y_{i}, z_{i}\right) \geq(\varepsilon, \varepsilon, \varepsilon) \gg 0$. A suitable choice of coefficients in the linear representations of functions $g^{r}$ yields a linear graph of $F$, with $F\left(x_{i}, z_{i}, t_{i}\right) \geq \varepsilon$ and unit MRC, for $\left(x_{i}, z_{i}, t_{i}\right) \geq(\varepsilon, \varepsilon, \varepsilon)$. As should be clear from footnote ${ }^{15}$ above, transferable transplants neither implies, nor is implied by, transferable utility.
} 


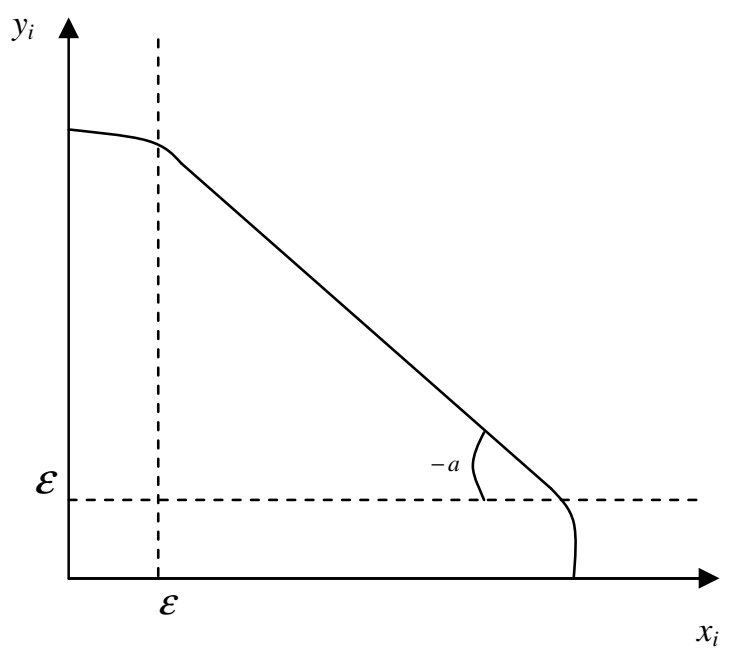

Fig 2.a

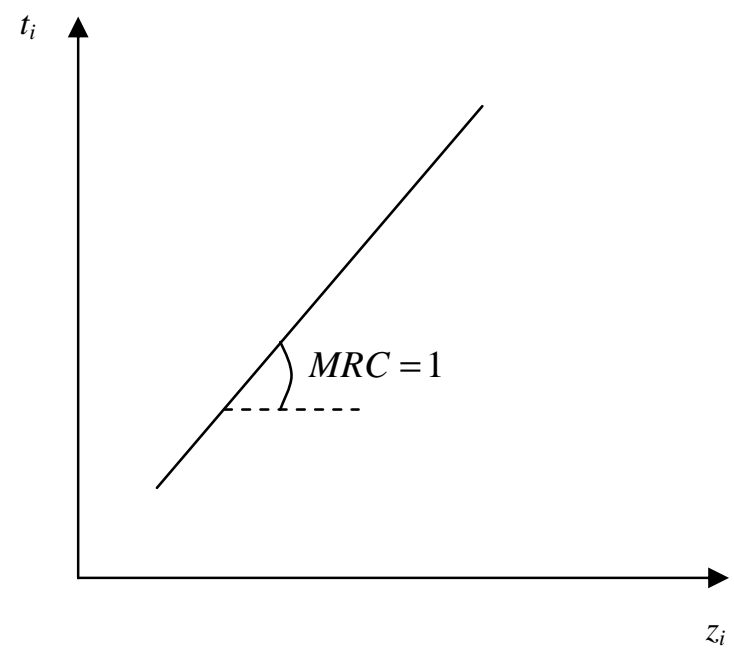

Fig 2.b 


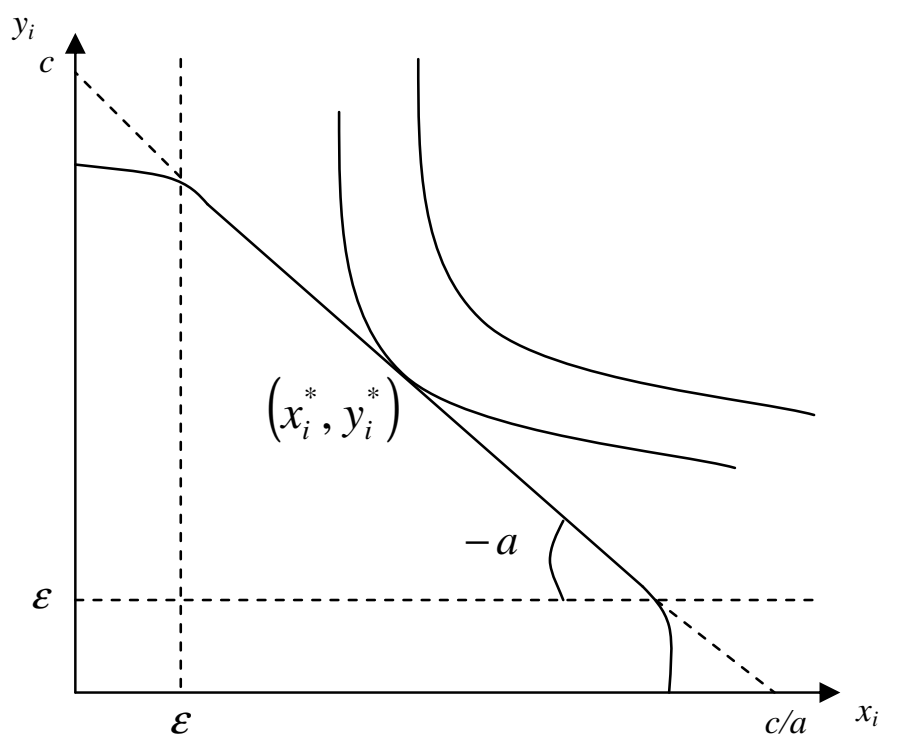

Fig 2.c

The first-order conditions of the second Lemma of the appendix (see Appendix: A-3) then readily imply that the agency's optimal distribution policy at the second stage of clear-sighted equilibrium is to transfer each hospital its own contribution when all hospitals contribute at least $\varepsilon$, that is, formally: the restriction of $\varphi^{0}$ to $\left\{z \in \mathbb{R}_{+}^{n}: \varepsilon \cdot e_{n} \leq z \leq \omega\right\}$ is the identity $z \rightarrow z .^{21}$ The first-order conditions of the third Lemma of the appendix (Appendix: A-3) then imply in turn that all hospitals have essentially the same set of optimal production combinations at the first stage of clear-sighted equilibrium, precisely: all elements of set $\left\{\left(x_{i}^{*}, y_{i}^{*}, z_{i}\right) \in \mathbb{R}_{+}^{3}: \varepsilon \leq z_{i} \leq \omega_{i}\right\}$, where $\left(x_{i}^{*}, y_{i}^{*}\right)$ is the same for all $i$ (but where, of course, $\omega_{i}$ may vary with $i$ ), solve hospital $i$ 's first stage program for all $z_{n / i} \geq \mathcal{E} \cdot e_{n-1}$. In other words, if all hospitals contribute at least $\varepsilon$, the agency's transfer policy makes each hospital's decision independent of others' decisions, and also makes hospital's final production of transplant and other care services independent of its own intermediary production of grafts for the agency; so that all hospitals end up choosing the same output combination for their final care services, and also end up indifferent to their intermediary graft production over range $\left[\varepsilon, \omega_{i}\right]$.

\footnotetext{
${ }^{21}$ Let $z_{i}=t_{i}$ and $\left(x_{i}, y_{i}\right)=\left(x_{i}^{*}, y_{i}^{*}\right)=\left(x^{*}, y^{*}\right)$ for all $i$ in the f.o.c., and recall the anonymity property of the social utility function implies that marginal social utilities of hospitals' utilities are equal whenever hospitals' utilities are equal.
} 
Let $\left(x_{i}^{*}, y_{i}^{*}\right)=\left(x^{*}, y^{*}\right)$. It clearly follows from definitions and paragraph above that any $\left(x^{*} \cdot e_{n}, y^{*} \cdot e_{n}, z\right)$ such that $\varepsilon . e_{n} \leq z \leq \omega$ is a clear-sighted equilibrium production of the medical care system, and that $t=z$ is the corresponding vector of equilibrium transfers. Note, finally, that: other equilibria might exist; $\left(x^{*} \cdot e_{n}, y^{*} \cdot e_{n}\right)$ is the unique socially efficient final production combination of the medical care, as a simple consequence of the first-order conditions of Theorem 2 and Corollary 1; and $z<\omega$ for all equilibria above except $\left(x^{*} \cdot e_{n}, y^{*} \cdot e_{n}, \omega\right)$. In particular, the marginal social utility of the potential graft resources of all hospitals is null in this example (while the marginal social utility of hospitals' aggregate contribution $\sum_{i \in N} z_{i}$ is positive). ${ }^{22}$ More precisely, some fraction of aggregate transplant resources does have a positive marginal social utility since hospitals and the agency have $u(0)$ and $W(u(0), \ldots, u(0))$ utility levels if $\sum_{i \in N} \omega_{i}=0$, but any $\omega \geq \varepsilon \cdot e_{n}$ suffices for sustaining the equilibria above. That is: transferable transplants technology applies if aggregate resources exceed threshold $n \mathcal{E}$ (technology being linear only for $\left(x_{i}, y_{i}, z_{i}, t_{i}\right) \geq(\varepsilon, \varepsilon, \varepsilon, \varepsilon)$ ); and this particular technology then makes any amount of aggregate transplant resources in excess of this threshold (any positive difference $\left.-n \varepsilon+\sum_{i \in N} \omega_{i}\right)$ socially useless.

\section{4-3--Monitored graft production}

A simple solution to the coordination problem raised in subsection 4-2 is the monitoring of graft production by the transplant agency. This solution actually appears trivial in the setup above, from the viewpoint of formal logic. It is interesting to develop because it captures, we believe, the organizational features of the Spanish transplant system that are at the origin of the latter's remarkable achievements analyzed in section 2 above.

The model is amended as follows. The transplant agency hires physicians and delegates them in hospitals in order to supervise graft production in each of them, with an objective of maximization of

${ }^{22}$ From the corollary of Theorem 2: $\delta_{i}=\partial_{i} W . \partial_{2} u . \partial_{3} F \cdot\left(1+\frac{\partial_{2} F}{\partial_{3} F}\right)=0$, while $\lambda=\partial_{i} W . \partial_{2} u . \partial_{3} F=\partial_{i} W . \partial_{2} u . c>0$, for all $i$. 
the latter subject to the legal, technical and endowment constraints detailed above. Formally, this new organizational trait amounts to letting the agency decide (through its delegates in hospitals) over hospitals' levels of graft production in $z=\left(z_{1}, \ldots, z_{n}\right)$. That is, the agency's monitoring opportunity set now reads $A_{0}^{M}(\omega)=\left\{\left(z, v^{z}, t\right) \in \mathbb{R}_{+}^{3 n}: \sum_{i \in N} t_{i} \leq \sum_{i \in N} z_{i} ; z_{i} \leq g^{z}\left(t_{i}, v_{i}^{z}, v_{i}^{x}+v_{i}^{y}+v_{i}^{z}\right)\right.$ and $z_{i} \leq \omega_{i}$ for all $\left.\mathrm{i}\right\}$, while its monitoring budget $B_{0}^{M}$ now covers the wages of supervisors in addition to the other costs of transplant provision. Similarly, Hospitals' monitored opportunity sets are defined as: $A_{i}^{M}\left(v_{i}^{z}, t_{i}\right)=\left\{\left(x_{i}, y_{i}, v_{i}^{x}, v_{i}^{y}\right) \in \mathbb{R}_{+}^{4}: x_{i} \leq g^{x}\left(t_{i}, v_{i}^{x}, v_{i}^{x}+v_{i}^{y}+v_{i}^{z}\right) ; y_{i} \leq g^{y}\left(t_{i}, v_{i}^{y}, v_{i}^{x}+v_{i}^{y}+v_{i}^{z}\right) ;\right.$ and $\left.v_{i}^{x}+v_{i}^{y} \leq B\right\}$

The public good externality between hospitals vanishes in this new specification of the transplant system, since it followed from their individual choice of a level of graft production, which now is essentially endorsed by the agency. The distinction between a myopic and a clear-sighted behaviour becomes pointless, consequently, in the sense that the (Nash non-cooperative) equilibrium of the transplant game is now independent of hospitals' understanding of agency's policy. The two-stage game may be specified, accordingly, as follows. Hospitals play second, each one solving $\max \left\{u\left(x_{i}, y_{i}\right):\left(x_{i}, y_{i}, v_{i}^{x}, v_{i}^{y}\right) \in A_{i}^{M}\left(v_{i}^{z}, t_{i}\right)\right\}$ with respect to $\left(x_{i}, y_{i}, v_{i}^{x}, v_{i}^{y}\right)$ for any given $\left(v_{i}^{z}, t_{i}\right)$. We denote by $\varphi_{i}^{M}$ hospital $i$ 's monitored reaction correspondence at this stage (solving program above for any $\left.\left(v_{i}^{z}, t_{i}\right)\right)$, and let $\varphi^{M}$ denote the associate product correspondence defined by $\varphi^{M}\left(v^{z}, t\right)=\left\{\left(x, y, v^{x}, v^{y}\right):\left(x_{i}, y_{i}, v_{i}^{x}, v_{i}^{y}\right) \in \varphi_{i}^{M}\left(v_{i}^{z}, t_{i}\right)\right.$ for all $\left.i\right\}$. The transplant agency plays in the first stage of the game, solving $\max \left\{W\left(u\left(x_{1}, y_{1}\right), \ldots, u\left(x_{n}, y_{n}\right)\right):\left(x, y, v^{x}, v^{y}\right) \in \varphi^{M}\left(v^{z}, t\right)\right.$ and $\left.\left(z, v^{z}, t\right) \in A_{0}^{M}(\omega)\right\} \quad$ with $\quad$ respect $\quad$ to $\left(z, v^{z}, t\right)$. An equilibrium of the game is a state $(x, y, z, v, t)$ that solves the latter program. We refer to this third notion of equilibrium as the monitored equilibrium.

We establish below that monitored equilibrium and social optimum coincide, provided that socially efficient production can be achieved by hospitals endowed with equal budgets $B$. This optimality property implies, in particular, in view of Theorem 2, that a monitored equilibrium exists, and that the corresponding socially optimal production of final care services is unique. 
Theorem 5: Suppose that, for any social optimum $(x, y, z, v, t)$, there exists a combination of general inputs $\tilde{v}$ such that $\tilde{v}_{i}^{x}+\tilde{v}_{i}^{y}=B$ and $\left(x_{i}, y_{i}, z_{i}\right)=g\left(t_{i}, \tilde{v}_{i}\right)$ for all $i$. Then, the monitored equilibrium is a social optimum.

Theorem 5 implies a clear advantage of monitored equilibrium, relative to clear-sighted equilibrium, in terms of the production of final care services of the medical care system. Optimizing the distribution of transplants does not suffice, in other words, for achieving socially efficient production. The latter supposes that some control be exerted also on graft production. This implies in turn some additional monitoring costs, captured in the simple model above through the (positive) difference $B_{0}^{M}-B_{0}$ between the agency's budgets in the monitored and clear-sighted games. A complete comparative evaluation of the two modes of regulation of the transplant care system supposes that their differences in terms of socially efficient production be balanced against their differences in terms of budgetary costs. The data collected in section 2 suggest that monitoring costs are actually low, relative to their remarkable impact on graft production. In other words, the Spanish experience shows a high productivity of monitoring expenses.

\section{Conclusion}

The economic organization of the transplant care system was characterized as a production economy of the public sector operating on the background of incomplete markets of inputs. The collection and circulation of transplants by the transplant agency induce public good interactions between hospitals. A socially optimal distribution policy of the agency cannot achieve alone the coordination of hospitals' production decisions at equilibrium and cannot in general attain alone the social optimum when potential graft resources are scarce, that is, equivalently, when the rationing constraints over the production of transplant inputs are binding at social optimum. Social optimum is attained by eliminating the public good interactions between hospitals through the optimal control of both the 
distribution and the production of transplant inputs by the agency. The data suggest that more than one half of Spain's donor rate differential with other countries proceeds from an adequate management of this public good problem by its national transplant organization. Improving the coordination of hospitals' production of transplants seems the principal and most efficient way for improving national donor rates. The other major way consists of lowering donation refusal rates through adequate exhortation policies and an adequate management of donation interviews.

\section{Appendix}

\section{A-1: Hospital's production possibility frontier}

Lemma 1: There exists a function $F$ such that, for any $\left(z_{i}, t_{i}\right) \in\left[0, \omega_{i}\right] \times \mathbb{R}_{++}$, the set of technically $\begin{array}{lllll}\text { accessible } & \text { output } & \text { of } & \text { hospital }\end{array}$ $\left\{\left(x_{i}, y_{i}, z_{i}\right) \in \mathbb{R}_{+}^{3}: x_{i} \leq g^{x}\left(t_{i}, B, B+\left(g_{B}^{z}\right)^{-1}\left(z_{i}\right)\right), y_{i} \leq F\left(x_{i}, z_{i}, t_{i}\right)\right.$ and $\left.z_{i} \leq \omega_{i}\right\}$, where $\left(g_{B}\right)^{-1}$ denotes the inverse of increasing partial function $v_{i}^{z} \rightarrow g^{z}\left(t_{i}, v_{i}^{z}, B+v_{i}^{z}\right)$. Function $F$ is defined over sets $\left\{\left(x_{i}, z_{i}, t_{i}\right) \in \mathbb{R}_{+}^{2} \times \mathbb{R}_{++}: x_{i} \leq g^{x}\left(t_{i}, B, B+v_{i}^{z}\right) ; z_{i} \leq g^{z}\left(t_{i}, v_{i}^{z}, B+v_{i}^{z}\right) ; v_{i}^{z} \geq 0\right\}$, and $C^{2}$ in the (non-empty) intersection of these convex domains with $\mathbb{R}_{++}^{3}$. It is decreasing and concave in $x_{i}$, decreasing in $z_{i}$ and increasing in $t_{i}$. Its partial derivatives read: $\partial_{1} F=-\frac{\partial_{2} g^{y}}{\partial_{2} g^{x}}$, $\partial_{2} F=\frac{1}{\partial_{2} g^{z}+\partial_{3} g^{z}}\left(\frac{\partial_{3} g^{x}}{\partial_{2} g^{x}} \partial_{2} g^{y}+\partial_{3} g^{y}\right)$, and $\partial_{3} F=\frac{\partial_{1} g^{x}}{\partial_{2} g^{x}} \partial_{2} g^{y}$ where the partial derivatives of $F$, $g^{x}, g^{y}$ and $g^{z}$ are respectively evaluated at $\left(x_{i}, z_{i}, t_{i}\right),\left(t_{i}, v_{i}^{x}, s\right),\left(t_{i}, B-v_{i}^{x}, s\right)$ and $\left(t_{i},\left(g_{B}^{z}\right)^{-1}\left(z_{i}\right), s\right)$ such that $s=B+\left(g_{B}^{z}\right)^{-1}\left(z_{i}\right)$ and $x_{i}=g^{x}\left(t_{i}, v_{i}^{x}, s\right)$.

Proof: Let $G^{r}:\left(t_{i}, v_{i}^{r}, z_{i}\right) \rightarrow g^{r}\left(t_{i}, v_{i}^{r}, B+\left(g_{B}^{z}\right)^{-1}\left(z_{i}\right)\right), r \in\{x, y\}$. Function $G^{x}$, being increasing in $v_{i}^{x}$ in $\mathbb{R}_{++}^{2} \times \mathbb{R}_{+}$(see Assumption 1), then admits a partial inverse relative to this variable, that is, there exists a function $h^{x}$ such that $g^{x}\left(t_{i}, h^{x}\left(x_{i}, z_{i}, t_{i}\right), B+\left(g_{B}^{z}\right)^{-1}\left(z_{i}\right)\right)=x_{i} \quad$ for all 
$\left(x_{i}, z_{i}, t_{i}\right) \in\left\{\left(x_{i}, z_{i}, t_{i}\right) \in \mathbb{R}_{++} \times \mathbb{R}_{+} \times \mathbb{R}_{++}: x_{i} \leq g^{x}\left(t_{i}, B, B+v_{i}^{z}\right) ; z_{i} \leq g^{z}\left(t_{i}, v_{i}^{z}, B+v_{i}^{z}\right) ; v_{i}^{z} \geq 0\right\}$. This domain of $h^{x}$ is convex and has a non-empty intersection with $\mathbb{R}_{++}^{3}$ by Assumption 1. The implicit function theorem moreover implies that $h^{x}$ is $C^{2}$ in the intersection of its domain with $\mathbb{R}_{++}^{3}$, with: $\partial_{1} h^{x}=1 / \partial_{2} g^{x}, \quad \partial_{2} h^{x}=-\partial_{3} g^{x} /\left(\partial_{2} g^{x}\left(\partial_{2} g^{z}+\partial_{3} g^{z}\right)\right)$ and $\partial_{3} h^{x}=-\partial_{1} g^{x} / \partial_{2} g^{x}$, where the partial derivatives of $h^{x}, g^{x}$ and $g^{z}$ are respectively evaluated at $\left(x_{i}, z_{i}, t_{i}\right),\left(t_{i}, h^{x}\left(x_{i}, z_{i}, t_{i}\right), B+\left(g_{B}^{z}\right)^{-1}\left(z_{i}\right)\right)$ and $\left(t_{i},\left(g_{B}^{z}\right)^{-1}\left(z_{i}\right), B+\left(g_{B}^{z}\right)^{-1}\left(z_{i}\right)\right)$. And $h^{x}$ is: increasing and convex in $x_{i}$ as inverse of increasing concave partial functions $v_{i}^{x} \rightarrow g^{x}\left(t_{i}, v_{i}^{x}, B+\left(g_{B}^{z}\right)^{-1}\left(z_{i}\right)\right)$; increasing in $z_{i}$ and decreasing in $t_{i}$ by the derivatives calculated above and Assumption 1. We may let $F$ be defined by: $F\left(x_{i}, z_{i}, t_{i}\right)=G^{y}\left(t_{i}, B-h^{x}\left(x_{i}, z_{i}, t_{i}\right), z_{i}\right)$ if $x_{i}>0 ; \quad F\left(x_{i}, z_{i}, t_{i}\right)=G^{y}\left(t_{i}, B, z_{i}\right)$ if $x_{i}=0$. One verifies immediately that $F$ is concave, decreasing in $x_{i}$ and in $z_{i}$, increasing in $t_{i}$. Its restriction to $\mathbb{R}_{++}^{3}$ is $C^{2}$, and $\partial_{1} F=-\frac{\partial_{2} g^{y}}{\partial_{2} g^{x}}, \partial_{2} F=\frac{1}{\partial_{2} g^{z}+\partial_{3} g^{z}}\left(\frac{\partial_{3} g^{x}}{\partial_{2} g^{x}} \partial_{2} g^{y}+\partial_{3} g^{y}\right)$, and $\partial_{3} F=\frac{\partial_{1} g^{x}}{\partial_{2} g^{x}} \partial_{2} g^{y}$ where the partial derivatives of $F, g^{x}$ and $g^{y}$ are respectively evaluated at $\left(x_{i}, z_{i}, t_{i}\right)$, $\left(t_{i}, h^{x}\left(x_{i}, z_{i}, t_{i}\right), B+\left(g_{B}^{z}\right)^{-1}\left(z_{i}\right)\right)$ and $\left(t_{i}, B-h^{x}\left(x_{i}, z_{i}, t_{i}\right), B+\left(g_{B}^{z}\right)^{-1}\left(z_{i}\right)\right)$

\section{A-2: Autarkic equilibrium, social optimum}

The proofs of this subsection are simple applications of standard techniques of convex optimization (Arrow and Enthoven (1961)).

Proof of Theorem 1: The existence of $\left(x_{i}^{*}, y_{i}^{*}, z_{i}^{*}, v_{i}^{*}, t_{i}^{*}\right)$ is a simple consequence of the continuity of hospital's utility function, maximized in non-empty compact set $\left\{\left(x_{i}, y_{i}, z_{i}, v_{i}, t_{i}\right) \in \mathbb{R}_{+}^{7}:\left(x_{i}, y_{i}, z_{i}\right) \leq g\left(t_{i}, v_{i}\right), t_{i} \leq z_{i}\right.$, and $\left.v_{i}^{x}+v_{i}^{y}+v_{i}^{z} \leq B\right\}$ (see Assumptions 1 and 2). The solutions are $\gg 0$ by Assumptions 1 and $2\left(\mathrm{a}>u(0)\right.$ utility suppose a $\gg 0$ production $\left(x_{i}, y_{i}\right)$ of final care services by Assumption 2, which implies in turn a $\gg 0$ vector $\left(z_{i}, v_{i}, t_{i}\right)$ of intermediary graft production and inputs by Assumption 1). The uniqueness of the optimal final production of care 
services $\left(x_{i}^{*}, y_{i}^{*}\right)$ follows from the strict quasi-concavity of utility function in $\mathbb{R}_{++}^{2}$ and convexity of opportunity set $\left\{\left(x_{i}, y_{i}, z_{i}, v_{i}, t_{i}\right) \in \mathbb{R}_{+}^{7}:\left(x_{i}, y_{i}, z_{i}\right) \leq g\left(t_{i}, v_{i}\right), t_{i} \leq z_{i}\right.$, and $\left.v_{i}^{x}+v_{i}^{y}+v_{i}^{z} \leq B\right\} \quad$ the latter implied by the concavity of production functions $\left.g^{r}, r \in\{x, y, z\}\right)$. "Unconstrained" program $\max \left\{u\left(x_{i}, y_{i}\right):\left(x_{i}, y_{i}, z_{i}, v_{i}, t_{i}\right) \geq 0,\left(x_{i}, y_{i}, z_{i}\right) \leq g\left(t_{i}, v_{i}\right), t_{i} \leq z_{i}\right.$, and $\left.v_{i}^{x}+v_{i}^{y}+v_{i}^{z} \leq B\right\}$ being identical for all $i$, so is the solution $\left(x_{i}^{*}, y_{i}^{*}, z_{i}^{*}, v_{i}^{*}, t_{i}^{*}\right)$. If $\left(x_{i}^{*}, y_{i}^{*}, z_{i}^{*}, v_{i}^{*}, t_{i}^{*}\right)$ verifies rationing constraint $z_{i} \leq \omega_{i}^{*}$ with a strict inequality, that is, if $z_{i}^{*}<\omega_{i}^{*}$, then it solves "constrained" program $\max \left\{u\left(x_{i}, y_{i}\right):\left(x_{i}, y_{i}, z_{i}, v_{i}, t_{i}\right) \geq 0,\left(x_{i}, y_{i}, z_{i}\right) \leq g\left(t_{i}, v_{i}\right), t_{i} \leq z_{i} \leq \omega_{i}^{*}\right.$, and $\left.v_{i}^{x}+v_{i}^{y}+v_{i}^{z} \leq B\right\} \quad$ as a simple consequence of definitions. The Kuhn and Tucker first-order conditions (f.o.c.) for the constrained program characterize $\left(x_{i}^{*}, y_{i}^{*}, z_{i}^{*}, v_{i}^{*}, t_{i}^{*}\right)$ by Arrow and Enthoven (1961: Theorems 1 and 2$)^{23}$. They read as follows: (i) $v_{i}^{x^{*}}+v_{i}^{y^{*}}+v_{i}^{z^{*}}=B$; (ii) $\left(x_{i}^{*}, y_{i}^{*}, z_{i}^{*}\right)=g\left(t_{i}^{*}, v_{i}^{*}\right)$; (iii) $\frac{\partial_{1} u}{\partial_{2} u}=\frac{\partial_{2} g^{y}}{\partial_{2} g^{x}} \quad$ (evaluated at $\left.\left(x_{i}^{*}, y_{i}^{*}, z_{i}^{*}, v_{i}^{*}, t_{i}^{*}\right)\right)$; (iv) and there exists $\gamma_{i} \in \mathbb{R}_{+}$such that $\gamma_{i} \cdot\left(\omega_{i}-z_{i}^{*}\right)=0$. Since $z_{i}^{*}<\omega_{i}^{*}$ by assumption, we must have $\gamma_{i}=0$ in the Kuhn and Tucker conditions, that is, $\gamma_{i}$ is determinate and $=0$ in the f.o.c., and interprets then as the hospital $i$ 's marginal utility of potential graft resources. The same applies to any hospital $j$ such that $\omega_{j} \geq \omega_{i}^{*}$. Finally, hospital's budget constraints are binding at autarkic equilibrium as a simple consequence of Assumption 1-(vi) and increasing utility, that is, these assumptions straightforwardly imply that any ceteris paribus increase in hospital's budget increases its maximal utility level in associate autarkic opportunity set.

Proof of Theorem 2: The social opportunity set $\left\{(x, y, z, v, t) \in \mathbb{R}_{+}^{7 n}: \sum_{i \in N} t_{i} \leq \sum_{i \in N} z_{i}\right.$; $z \leq \omega ; \sum_{i \in N} v_{i}^{x}+v_{i}^{y} \leq n B$; and $\left(x_{i}, y_{i}, z_{i}\right) \leq g\left(t_{i}, v_{i}\right)$ for all i $\}$ is non-empty (it contains 0 ), compact (by continuity of $g$ ) and convex (by concavity of $g$ ). It has a non-empty intersection with $\mathbb{R}_{++}^{7 n}$ by our assumptions relative to the productivity of hospital's technology (see Assumption 1, notably parts (iv),

\footnotetext{
${ }^{23}$ These theorems of Arrow and Enthoven apply here, in spite of the non-differentiability of objective and constraint functions on the lower boundary of their domains (the boundary conditions of Assumptions 1, 2 and 3 ), because these functions admit quasi-concave differentiable extensions with same solutions of the program. The same remark applies to our use of the first-order conditions of Mas-Colell (1985): D.3.3 in Lemma 3 below: the objective and constraint functions of the program admit $C^{1}$ extensions with same solutions of the program.
} 
(v) and (vi)). The continuity of social utility function $(x, y) \rightarrow W\left(u\left(x_{1}, y_{1}\right), \ldots, u\left(x_{n}, y_{n}\right)\right)$ therefore implies the existence of a social optimum $\left(x^{*}, y^{*}, z^{*}, v^{*}, t^{*}\right)$, which must be $\gg 0$ by Assumption 1 and the boundary conditions of Assumption 2 and 3. The optimal production of final care services $\left(x^{*}, y^{*}\right)$ is unique by the strict quasi-concavity of $(x, y) \rightarrow W\left(u\left(x_{1}, y_{1}\right), \ldots, u\left(x_{n}, y_{n}\right)\right)$ in $\mathbb{R}_{++}^{2 n}$ and convexity of the social opportunity set. The Kuhn and Tucker first-order conditions are necessary and sufficient at a $\gg 0$ solution of convex program $\max \left\{W\left(u\left(x_{1}, y_{1}\right), \ldots, u\left(x_{n}, y_{n}\right)\right):(x, y, z, v, t) \geq 0 ; \sum_{i \in N} t_{i} \leq \sum_{i \in N} z_{i}\right.$; $z \leq \omega^{\prime} ; \sum_{i \in N} v_{i}^{x}+v_{i}^{y} \leq n B$; and $\left(x_{i}, y_{i}, z_{i}\right) \leq g\left(t_{i}, v_{i}\right)$ for all $\left.i\right\}$ by Arrow and Enthoven (1961: Theorems 1 and 2). Strictly increasing utility and production functions in the positive orthant readily imply that constraints $\sum_{i \in N} t_{i} \leq \sum_{i \in N} z_{i}$ and $\left(x_{i}, y_{i}, z_{i}\right) \leq g\left(t_{i}, v_{i}\right)$ are binding, with positive associate multipliers, in the f.o.c.. Strictly increasing utility and Assumption 1-(vi) moreover imply that aggregate budget constraint $\sum_{i \in N} v_{i}^{x}+v_{i}^{y} \leq n B$ is also binding, with a positive associate multiplier, at social optimum. These remarks and some calculations yield the following system of characterizing f.o.c., where partial derivatives are evaluated at the optimum: (i) $\sum_{i \in N} t_{i}^{*}=\sum_{i \in N} z_{i}^{*}$; (ii) $\sum_{i \in N} v_{i}^{x^{*}}+v_{i}^{y^{*}}=n B$; (iii) for all $i, \quad\left(x_{i}^{*}, y_{i}^{*}, z_{i}^{*}\right)=g\left(t_{i}^{*}, v_{i}^{*}\right)$ and $\frac{\partial_{1} u}{\partial_{2} u}=\frac{\partial_{2} g^{y}}{\partial_{2} g^{x}} ;$ (iv) and there exists $(\lambda, \delta) \in \mathbb{R}_{++} \times \mathbb{R}_{+}^{n}$ such that $\partial_{i} W . \partial_{1} u . \partial_{1} g^{x}=\lambda, \quad \delta_{i}=\lambda+\frac{\partial_{i} W}{\partial_{2} g^{z}+\partial_{3} g^{z}} .\left(\partial_{1} u . \partial_{3} g^{x}+\partial_{2} u . \partial_{3} g^{y}\right)<\lambda, \quad$ and $\delta_{i} \cdot\left(\omega_{i}-z_{i}^{*}\right)=0$ for all $i$, where $\lambda$ is the multiplier associated with constraint $\sum_{i \in N} t_{i} \leq \sum_{i \in N} z_{i}$. Finally, the characterization of scarcity in the last part of Theorem 2 is a simple consequence of definitions and the characterizing f.o.c. above.

\section{A-3: Agents' behaviour at clear-sighted equilibrium}

Lemma 2: Agency's transfer policy: The agency's transfer correspondence at the second stage of the clear-sighted game identifies with a continuous function $\varphi^{0}:\left\{z \in \mathbb{R}^{n}: 0 \leq z \leq \omega\right\} \rightarrow \mathbb{R}_{+}^{n}$ such that $\varphi^{0}(0)=0$ and $\varphi^{0}(z) \gg 0$ for all $z>0$. Its restriction to $\left\{z \in \mathbb{R}_{+}^{n}: 0<z \leq \omega\right\}$ solves, for any given $z$, 
the following system of first-order conditions in $(x, t):$ (i) $\sum_{i \in N} t_{i}=\sum_{i \in N} z_{i}$; (ii) for all $i$, $\frac{\partial_{1} u\left(x_{i}, F\left(x_{i}, z_{i}, t_{i}\right)\right)}{\partial_{2} u\left(x_{i}, F\left(x_{i}, z_{i}, t_{i}\right)\right)}=-\partial_{1} F\left(x_{i}, z_{i}, t_{i}\right)$; (iii) and there exists $\lambda \in \mathbb{R}_{++}$such that, for all $i$, $\partial_{i} W\left(u\left(x_{1}, F\left(x_{1}, z_{1}, t_{1}\right)\right), \ldots, u\left(x_{n}, F\left(x_{n}, z_{n}, t_{n}\right)\right)\right) . \partial_{2} u\left(x_{i}, F\left(x_{i}, z_{i}, t_{i}\right)\right) . \partial_{3} F\left(x_{i}, z_{i}, t_{i}\right)=\lambda$

Proof: Sets of alternatives $A_{i}\left(t_{i}, \omega_{i}\right)$ and $A_{0}(z)$ being non-empty, compact and convex for all nonnegative $(t, z)$ such that $z \leq \omega$, and the agency's utility function being continuous, program $\max \left\{W\left(u\left(x_{1}, y_{1}\right), \ldots, u\left(x_{n}, y_{n}\right)\right):\left(x_{i}, y_{i}, z_{i}, v_{i}\right) \in A_{i}\left(t_{i}, \omega_{i}\right)\right.$ for all $i$, and $\left.t \in A_{0}(z)\right\} \quad$ has one solution $(x, y, t)$ at least, for any fixed non-negative $z \leq \omega$. That is, correspondence $\varphi^{0}:\left\{z \in \mathbb{R}^{n}: 0 \leq z \leq \omega\right\} \rightarrow \mathbb{R}_{+}^{n}$ is well-defined (i.e. has non-empty values over its domain). Its values are compact by continuity of $u$, and convex by convexity of $\left\{(x, y):\left(x_{i}, y_{i}, z_{i}, v_{i}\right) \in A_{i}\left(t_{i}, \omega_{i}\right)\right.$ for all $i$, and $\left.t \in A_{0}(z)\right\}$ and quasi-concavity of $u$.

$A_{0}(0)=\{0\} \quad$ by $\quad$ definitions, $\quad A_{i}\left(0, \omega_{i}\right)=\left\{\left(0, y_{i}, z_{i}, v_{i}\right) \in \mathbb{R}_{+}^{6}: y_{i} \leq\right.$ $g^{y}\left(0, v_{i}^{y}, v_{i}^{x}+v_{i}^{y}+v_{i}^{z}\right), z_{i} \leq g^{z}\left(0, v_{i}^{z}, v_{i}^{x}+v_{i}^{y}+v_{i}^{z}\right), z_{i} \leq \omega_{i}$ and $\left.v_{i}^{x}+v_{i}^{y} \leq B\right\}$ for all $i$ by definitions and Assumption 1-(iii). These facts and Assumptions 1 and 2 imply that the set of solutions of the agency's program when $z=0$ coincides with the corresponding set of alternatives of the agency's program, that is, with set $\left\{(x, y, t): x=t=0\right.$ and $0 \leq y_{i} \leq g^{y}(0, B, B)$ for all $\left.i\right\}$, the agency's and hospitals' utilities being then $=W(0)$ over this whole set. In particular: $\varphi^{0}(0)=\{0\}$.

Suppose from now on that $0<z \leq \omega$.

$A_{0}(z) \cap \mathbb{R}_{++}^{n}$ is non-empty whenever $z>0$, that is, it is always possible for the agency to make positive transplant transfers to all hospitals whenever some $>0$ quantity of transplant is available. The agency's set of alternatives $\left\{(x, y, v, t) \in \mathbb{R}_{+}^{7 n}:\left(x_{i}, y_{i}, z_{i}, v_{i}\right) \in A_{i}\left(t_{i}, \omega_{i}\right)\right.$ for all $i$, and $\left.t \in A_{0}(z)\right\}$ is convex for all $z$, by the concavity of production functions $g^{r}, r \in\{x, y, z\}$ (see Assumption 1). The boundary conditions of Assumptions 2 and 3 relative to utility functions, the strict quasi-concavity of the social utility function in $\mathbb{R}_{++}^{2 n}$, and Assumption 1 then readily imply that the solutions of the agency's program are $\gg 0$ vectors $(x, y, v, t)$, which moreover imply a unique optimal production of final care 
services $(x, y)$, whenever $z>0$. From Lemma 1 and strictly increasing hospital's utility, such interior solutions can be characterized, equivalently, as interior solutions to : $\max \left\{W\left(u\left(x_{1}, F\left(x_{1}, z_{1}, t_{1}\right)\right), \ldots, u\left(x_{n}, F\left(x_{n}, z_{n}, t_{n}\right)\right)\right): 0 \leq x_{i} \leq g^{x}\left(t_{i}, B, B+\left(g_{B}^{z}\right)^{-1}\left(z_{i}\right)\right)\right.$, and $\left.t \in A_{0}(z)\right\}$ where "interior" now means either that $(x, y, t) \gg 0$ or, equivalently, that $t \gg 0$ and $0<x_{i}<g^{x}\left(t_{i}, B, B+\left(g_{B}^{z}\right)^{-1}\left(z_{i}\right)\right)$ for all $i$. The necessary first-order conditions (f.o.c.) for the latter $C^{2}$ program read as follows (e.g. Mas-Colell (1985): D.3.3): (i) $\sum_{i \in N} t_{i} \leq \sum_{i \in N} z_{i}$; (ii) for all $i$, $\frac{\partial_{1} u\left(x_{i}, F\left(x_{i}, z_{i}, t_{i}\right)\right)}{\partial_{2} u\left(x_{i}, F\left(x_{i}, z_{i}, t_{i}\right)\right)}=-\partial_{1} F\left(x_{i}, z_{i}, t_{i}\right)$; (iii) and there exists a $\geq 0$ real number $\lambda$ such that $\partial_{i} W\left(u\left(x_{1}, F\left(x_{1}, z_{1}, t_{1}\right)\right), \ldots, u\left(x_{n}, F\left(x_{n}, z_{n}, t_{n}\right)\right)\right) . \partial_{2} u\left(x_{i}, F\left(x_{i}, z_{i}, t_{i}\right)\right) . \partial_{3} F\left(x_{i}, z_{i}, t_{i}\right)=\lambda \quad$ and $\lambda\left(\sum_{i \in N} z_{i}-\sum_{i \in N} t_{i}\right)=0$ for all $i$

Utility functions being strictly increasing in the positive orthant (see Assumptions 2 and 3) and function $F$ being strictly increasing relative to $t_{i}>0$ in $\mathbb{R}_{++}^{3}$ (see Lemma 1 ), the third part of the f.o.c. readily implies that $\lambda>0$ and $\sum_{i \in N} t_{i}=\sum_{i \in N} z_{i}$, that is, the agency's marginal utility of aggregate graft provision is $>0$ and aggregate graft production is entirely transferred to hospitals at agency's optimum.

The non-convex $\quad \max \left\{W\left(u\left(x_{1}, F\left(x_{1}, z_{1}, t_{1}\right)\right), \ldots, u\left(x_{n}, F\left(x_{n}, z_{n}, t_{n}\right)\right)\right): 0 \leq x_{i} \leq\right.$ $g^{x}\left(t_{i}, B, B+\left(g_{B}^{z}\right)^{-1}\left(z_{i}\right)\right)$, and $\left.t \in A_{0}(z)\right\} \quad$ being equivalent to the convex program $\max \left\{W\left(u\left(x_{1}, y_{1}\right), \ldots, u\left(x_{n}, y_{n}\right)\right):\left(x_{i}, y_{i}, z_{i}, v_{i}\right) \in A_{i}\left(t_{i}, \omega_{i}\right)\right.$ for all $i$,and $\left.t \in A_{0}(z)\right\}$, the necessary f.o.c. above are also necessary first-order conditions for the latter. And the f.o.c. of program $\max \left\{W\left(u\left(x_{1}, y_{1}\right), \ldots, u\left(x_{n}, y_{n}\right)\right):\left(x_{i}, y_{i}, z_{i}, v_{i}\right) \in A_{i}\left(t_{i}, \omega_{i}\right)\right.$ for all $i$, and $\left.t \in A_{0}(z)\right\} \quad$ are also sufficient conditions for an interior solution of the latter by Arrow and Enthoven (1961: Theorem 1). They characterize, therefore, the solutions whenever $z>0$.

Let us prove, to finish with, that $\varphi^{0}$ is single-valued and continuous over $\left\{z \in \mathbb{R}_{+}^{n}: z \leq \omega\right\}$.

We already proved that $\varphi^{0}(0)=\{0\}$. Let $z>0$ and $\left(x^{*}, y^{*}, t^{*}\right)$ solve $\max \left\{W\left(u\left(x_{1}, F\left(x_{1}, z_{1}, t_{1}\right)\right), \ldots, u\left(x_{n}, F\left(x_{n}, z_{n}, t_{n}\right)\right)\right): 0 \leq x_{i} \leq g^{x}\left(t_{i}, B, B+\left(g_{B}^{z}\right)^{-1}\left(z_{i}\right)\right) ; t \in A_{0}(z)\right\} . \quad$ We 
established above that optimal $(x, y)$ is unique, $=\left(x^{*}, y^{*}\right)$, and that $t^{*} \gg 0$. Function $F$ being increasing in transfer, $t_{i}^{*}$ is necessarily unique for all $i$, as unique solution of equation in $t_{i}$ : $y_{i}^{*}=F\left(x_{i}^{*}, z_{i}, t_{i}\right)$. Therefore, $\varphi^{0}$ is single-valued over $\left\{z \in \mathbb{R}_{+}^{n}: z \leq \omega\right\}$. It identifies, in other words, with a function $\left\{z \in \mathbb{R}_{+}^{n}: z \leq \omega\right\} \rightarrow \mathbb{R}_{+}^{n}$ over this domain.

Let sequence $\left(z^{q}\right)_{q \in \mathbb{N}}$ of elements of $\left\{z \in \mathbb{R}_{+}^{n}: z \leq \omega\right\}$ converge to $z^{*}$. Suppose first that $z^{*}>0$. Then $\varphi^{0}\left(z^{*}\right) \gg 0$ and there exists $q_{0} \in \mathbb{N}$ such that $z^{q}>0$ and $\varphi^{0}\left(z^{q}\right) \gg 0$ for all $q \geq q_{0}$. Therefore, $z^{*}$ and all $z^{q}$ such that $q \geq q_{0}$ verify the system of $C^{1}$ f.o.c. above. $\left(\varphi^{0}\left(z^{q}\right)\right)_{q \geq q_{0}}$, being a sequence of elements of compact set $\left\{t \in \mathbb{R}_{+}^{n}: \sum_{i \in N} t_{i} \leq 1\right\}$, has at least one limit point $t^{*}$ in that set. $t^{*}$ verifies the f.o.c. at $z^{*}$ by continuity of the latter. Therefore $t^{*}=\varphi^{0}\left(z^{*}\right)$, and continuity in $\left\{z \in \mathbb{R}_{+}^{n}: 0<z \leq \omega\right\}$ is established. Suppose, finally, that $z^{*}=0$. By definition of $\varphi^{0}, \varphi^{0}\left(z^{q}\right)$ is $\geq 0$ and verifies inequalities $0 \leq \sum_{i \in N} \varphi_{i}^{0}\left(z^{q}\right) \leq \sum_{i \in N} z_{i}^{q}$ for all $q$. Therefore $\lim _{z^{q} \rightarrow 0, z^{q} \geq 0} \varphi^{0}\left(z^{q}\right)$ is well-defined, $=0=\varphi^{0}(0)$, and continuity at 0 is established.

Lemma 3: Hospital's behaviour: Hospital $i$ 's reaction correspondence at the first stage of the clearsighted game is a well-defined, upper hemi-continuous correspondence $\varphi_{i}^{C}: z \in\left\{z \in \mathbb{R}_{+}^{n}: z \leq \omega\right\} \rightarrow \mathbb{R}_{+}^{6}$ such that: $\varphi_{i}^{C}(z) \subset \mathbb{R}_{++}^{6}$ whenever $z_{n / i}=0 ; \quad \varphi_{i}^{C}(z) \subset \mathbb{R}_{++}^{2} \times \mathbb{R}_{+} \times \mathbb{R}_{++}^{2} \times \mathbb{R}_{+}$whenever $z_{n / i} \neq 0$. Let $\tilde{z} \in\left\{z \in \mathbb{R}_{+}^{n-1}: z \leq \omega\right\}$ be fixed, $\left(x_{i}^{*}, y_{i}^{*}, z_{i}^{*}, v_{i}^{*}\right) \in \varphi_{i}^{C}(\tilde{z})$ be such that $z_{i}^{*}>0$, and suppose that $z_{i} \rightarrow \varphi_{i}^{0}\left(\left(\tilde{z}_{n / i}, z_{i}\right)\right)$ is $C^{1}$ in some interval open in $\left(0, \omega_{i}\right]$ containing $z_{i}^{*}$. Then, $\left(x_{i}^{*}, y_{i}^{*}, z_{i}^{*}, v_{i}^{*}\right)$ verifies the following system of first-order conditions: (i) $y_{i}^{*}=F\left(x_{i}^{*}, z_{i}^{*}, \varphi_{i}^{0}\left(\left(\tilde{z}_{n / i}, z_{i}^{*}\right)\right)\right)$; $\left(x_{i}^{*}, y_{i}^{*}, z_{i}^{*}\right)=g\left(\varphi_{i}^{0}\left(\left(\tilde{z}_{n l i}, z_{i}^{*}\right)\right), v_{i}^{*}\right)$; (iii) $\frac{\partial_{1} u\left(x_{i}^{*}, y_{i}^{*}\right)}{\partial_{2} u\left(x_{i}^{*}, y_{i}^{*}\right)}=-\partial_{1} F\left(x_{i}^{*}, z_{i}^{*}, \varphi_{i}^{0}\left(\left(\tilde{z}_{n l i}, z_{i}^{*}\right)\right)\right)$; (iv) and there exists $\delta_{i} \in \mathbb{R}_{+}$such that $\partial_{2} u\left(x_{i}^{*}, y_{i}^{*}\right) \cdot\left(\partial_{2} F\left(x_{i}^{*}, z_{i}^{*}, \varphi_{i}^{0}\left(\left(\tilde{z}_{n / i}, z_{i}^{*}\right)\right)\right)+\partial_{3} F\left(x_{i}^{*}, z_{i}^{*}, \varphi_{i}^{0}\left(\left(\tilde{z}_{n / i}, z_{i}^{*}\right)\right)\right) \cdot \partial_{i} \varphi_{i}^{0}\left(\left(\tilde{z}_{n / i}, z_{i}^{*}\right)\right)\right)=\delta_{i}$ and $\delta_{i}\left(\omega_{i}-z_{i}^{*}\right)=0$. If function $\left(x_{i}, z_{i}\right) \rightarrow u\left(x_{i}, F\left(x_{i}, z_{i}, \varphi_{i}^{0}\left(\left(\tilde{z}_{n / i}, z_{i}\right)\right)\right)\right)$ is, moreover, quasi-concave over $\left\{\left(x_{i}, z_{i}\right) \in \mathbb{R}_{+}^{2}: 0<z_{i} \leq \omega_{i}\right\}$, then, the first-order conditions above characterize the $\gg 0$ elements of 
$\varphi_{i}^{C}(\tilde{z})$, that is, $\left(x_{i}^{*}, y_{i}^{*}, z_{i}^{*}, v_{i}^{*}\right) \in \varphi_{i}^{C}(\tilde{z})$ and is $\gg 0$ if and only if $\left(x_{i}^{*}, y_{i}^{*}, z_{i}^{*}, v_{i}^{*}\right)$ verifies the f.o.c. and is $\gg 0$.

Proof: Note first that set $A_{i}\left(\varphi_{i}^{0}(z), \omega_{i}\right)$ being non-empty and compact for all $\left(z, \omega_{i}\right) \geq 0$ and utility function $u$ being continuous, program $\max \left\{u\left(x_{i}, y_{i}\right):\left(x_{i}, y_{i}, z_{i}, v_{i}\right) \in A_{i}\left(\varphi_{i}^{0}(z), \omega_{i}\right)\right\}$ has one solution at least for any fixed $\left(z, \omega_{i}\right) \geq 0$. Therefore, correspondence $\varphi_{i}^{C}: \mathbb{R}_{+}^{n+1} \rightarrow \mathbb{R}_{+}^{6}$ is well- defined.

Let $z_{n / i}=0$. We established in Lemma 2 that $\varphi^{0}(0)=0$ and $\varphi^{0}(z) \gg 0$ whenever $z>0$. And we supposed in Assumption 1 that $g^{x}\left(0, v_{i}\right)=0$ for all $v_{i}$. Therefore, hospital $i$ 's optimal graft production $z_{i}$ is positive, for then and only then is a $>u(0)$ utility level accessible for hospital $i$ by Assumption 2. In other words, if other hospitals contribute nothing, hospital $i$ is willing to contribute something, in order to receive some positive transfer from the agency that allows for a $\gg 0$ final production $\left(x_{i}, y_{i}\right)$ and $>u(0)$ utility.

Let $z_{n / i} \in\left\{z_{n / i} \in \mathbb{R}_{+}^{n-1}: z_{n / i} \leq \omega_{n / i}\right\}$ be fixed from there on. $\varphi^{0}\left(\left(z_{n / i}, z_{i}^{*}\right)\right) \gg 0$ for any optimal graft production $z_{i}^{*}$ of $\varphi_{i}^{C}(z)$ by the paragraph above, so that program $\max \left\{u\left(x_{i}, y_{i}\right):\left(x_{i}, y_{i}, z_{i}, v_{i}\right) \in A_{i}\left(\varphi_{i}^{0}\left(\left(z_{n / i}, z_{i}\right)\right), \omega_{i}\right)\right\} \quad$ can $\quad$ be rewritten equivalently as $\max \left\{u\left(x_{i}, F\left(x_{i}, z_{i}, \varphi_{i}^{0}\left(\left(z_{n / i}, z_{i}\right)\right)\right)\right): 0 \leq x_{i} \leq g^{x}\left(\varphi_{i}^{0}\left(\left(z_{n / i}, z_{i}\right)\right), B, B+\left(g_{B}^{z}\right)^{-1}\left(z_{i}\right)\right)\right.$, and $\left.0 \leq z_{i} \leq \omega_{i}\right\}$

by Lemma 1. Solutions in $\left(x_{i}, y_{i}\right)$ are "interior", that is, $\left(x_{i}, F\left(x_{i}, z_{i}^{*}, \varphi_{i}^{0}\left(\left(z_{n / i}, z_{i}^{*}\right)\right)\right)\right) \gg 0$, by the boundary condition of Assumption 2. But we may have a corner solution in $z_{i}$, that is, an optimal graft production $z_{i}^{*}$ equal to either 0 or $\omega_{i}\left(z_{i}^{*}=0\right.$ only if $\left.z_{n / i}>0\right)$.

Suppose that optimal graft production $z_{i}^{*}$ is positive, and that $z_{i} \rightarrow \varphi_{i}^{0}\left(\left(z_{n / i}, z_{i}\right)\right)$ is $C^{1}$ in an interval open in $\left(0, \omega_{i}\right]$ containing $z_{i}^{*}$. The necessary first-order conditions for solution $\left(x_{i}, z_{i}^{*}\right)$ of the reduced program above then read as follows (e.g. Mas-Colell (1985): D.1): $\frac{\partial_{1} u\left(x_{i}, F\left(x_{i}, z_{i}^{*}, \varphi_{i}^{0}\left(\left(z_{n / i}, z_{i}^{*}\right)\right)\right)\right)}{\partial_{2} u\left(x_{i}, F\left(x_{i}, z_{i}^{*}, \varphi_{i}^{0}\left(\left(z_{n / i}, z_{i}^{*}\right)\right)\right)\right)}=-\partial_{1} F\left(x_{i}, z_{i}^{*}, \varphi_{i}^{0}\left(\left(z_{n / i}, z_{i}^{*}\right)\right)\right)$; (ii) and there exists $\delta_{i} \in \mathbb{R}_{+}$such that $\partial_{2} u\left(x_{i}, y_{i}\right) .\left(\partial_{2} F\left(x_{i}, z_{i}^{*}, \varphi_{i}^{0}\left(\left(z_{n l i}, z_{i}^{*}\right)\right)\right)+\partial_{3} F\left(x_{i}, z_{i}^{*}, \varphi_{i}^{0}\left(\left(z_{n / i}, z_{i}^{*}\right)\right)\right) . \partial_{i} \varphi_{i}^{0}\left(\left(z_{n l i}, z_{i}^{*}\right)\right)\right)=\delta_{i}$ and $\delta_{i}\left(\omega_{i}-z_{i}^{*}\right)=0$.

Conversely, if $\left(x_{i}, z_{i}\right) \rightarrow u\left(x_{i}, F\left(x_{i}, z_{i}, \varphi_{i}^{0}\left(\left(z_{n / i}, z_{i}\right)\right)\right)\right)$ is quasi-concave over $\left\{\left(x_{i}, z_{i}\right) \in \mathbb{R}_{+}^{2}: 0<z_{i} \leq \omega_{i}\right\}$, 
if $z_{i} \rightarrow \varphi_{i}^{0}\left(\left(z_{n / i}, z_{i}\right)\right)$ is $C^{1}$ in an interval open in $\left(0, \omega_{i}\right]$ containing $z_{i}^{*}>0$, and if $\left(x_{i}, z_{i}^{*}\right) \gg 0$ verifies the f.o.c. above, then $\left(x_{i}^{*}, z_{i}^{*}\right)$ solves reduced program $\max \left\{u\left(x_{i}, F\left(x_{i}, z_{i}, \varphi_{i}^{0}\left(\left(z_{n / i}, z_{i}\right)\right)\right)\right): z_{i} \leq \omega_{i}\right\}$, by Arrow and Enthoven (1961: Theorem 1). Let us establish, finally, that $\varphi_{i}^{C}$ is upper hemi-continuous (u.h.c.) in $\left\{z \in \mathbb{R}_{+}^{n}: z \leq \omega\right\}$ for all $i$.

Let $\left(z^{q}\right)_{q \in \mathbb{N}}$ be a sequence of elements of $\left\{z \in \mathbb{R}_{+}^{n}: z \leq \omega\right\}$ converging to $z^{*}$, and sequence $\left(\left(x_{i}^{q}, y_{i}^{q}, \bar{z}_{i}^{q}, v_{i}^{q}\right)\right)_{q \in \mathbb{N}}$ be such that $\left(x_{i}^{q}, y_{i}^{q}, \bar{z}_{i}^{q}, v_{i}^{q}\right) \in \varphi_{i}^{C}\left(z^{q}\right)$ for all $q$ and converge to $\left(x_{i}^{*}, y_{i}^{*}, \bar{z}_{i}^{*}, v_{i}^{*}\right)$. We want to prove that $\left(x_{i}^{*}, y_{i}^{*}, \bar{z}_{i}^{*}, v_{i}^{*}\right) \in \varphi_{i}^{C}\left(z^{*}\right)$. Note that $\left(x_{i}^{*}, y_{i}^{*}, \bar{z}_{i}^{*}, v_{i}^{*}\right) \in A_{i}\left(\varphi_{i}^{0}\left(z^{*}\right), \omega_{i}\right)=$ $\left\{\left(x_{i}, y_{i}, z_{i}, v_{i}\right) \in \mathbb{R}_{+}^{6}: \quad\left(x_{i}, y_{i}, z_{i}\right) \leq g\left(\varphi_{i}^{0}\left(z^{*}\right), v_{i}\right), \quad z_{i} \leq \omega_{i}\right.$, and $\left.v_{i}^{x}+v_{i}^{y} \leq B\right\} \quad$ by continuity of $g$ and $\varphi_{i}^{0}$. Let $\left(\tilde{x}_{i}, \tilde{y}_{i}, \tilde{z}_{i}, \tilde{v}_{i}\right)$ be any element of $A_{i}\left(\varphi_{i}^{0}\left(z^{*}\right), \omega_{i}\right)$.

If $\tilde{x}_{i}$ or $\tilde{y}_{i}$ is $=0$, then $u\left(x_{i}^{*}, y_{i}^{*}\right) \geq u\left(\tilde{x}_{i}, \tilde{y}_{i}\right)=u(0)$ by Assumption 2 .

Suppose that $\left(\tilde{x}_{i}, \tilde{y}_{i}\right) \gg 0$. Note that, then, $\varphi_{i}^{0}\left(z^{*}\right)>0$ and $\tilde{x}_{i}<g^{x}\left(\varphi_{i}^{0}\left(z^{*}\right), B, B+\left(g_{B}^{z}\right)^{-1}\left(\tilde{z}_{i}\right)\right)$ by the definition of $A_{i}\left(\varphi_{i}^{0}\left(z^{*}\right), \omega_{i}\right)$ and Assumption 1. We construct a sequence $\left(\left(\tilde{x}_{i}^{q}, \tilde{y}_{i}^{q}, \tilde{z}_{i}^{q}, \tilde{v}_{i}^{q}\right)\right)_{q \in \mathbb{N}}$ that converges to $\left(\tilde{x}_{i}, \tilde{y}_{i}, \tilde{z}_{i}, \tilde{v}_{i}\right)$ and is such that $\left(\tilde{x}_{i}^{q}, \tilde{y}_{i}^{q}, \tilde{z}_{i}^{q}, \tilde{v}_{i}^{q}\right) \in A_{i}\left(\varphi_{i}^{0}\left(z^{q}\right), \omega_{i}\right)$ for all $q$. There exists $q_{0} \in \mathbb{N}$ such that $\varphi_{i}^{0}\left(z^{q}\right)>0$ and $\tilde{x}_{i}<g^{x}\left(\varphi_{i}^{0}\left(z^{q}\right), B, B+\left(g_{B}^{z}\right)^{-1}\left(\tilde{z}_{i}^{q}\right)\right)$ for all $q \geq q_{0}$, by continuity of $g^{x}$, $\varphi_{i}^{0}$ and $\left(g_{B}^{z}\right)^{-1}$. If $\tilde{y}_{i}<F\left(\tilde{x}_{i}, \tilde{z}_{i}, \varphi_{i}^{0}\left(z^{*}\right)\right)$, then, by construction of $F$ (see the proof of Lemma 1), either $\tilde{v}_{i}^{x}+\tilde{v}_{i}^{y}=B$ and $\tilde{y}_{i}<g^{y}\left(\varphi_{i}^{0}\left(z^{*}\right), \tilde{v}_{i}^{y}, \tilde{v}_{i}^{x}+\tilde{v}_{i}^{y}+\tilde{v}_{i}^{z}\right)$ or $\tilde{v}_{i}^{x}+\tilde{v}_{i}^{y}<B$; therefore, by continuity of $F, \varphi_{i}^{0}$ and $g$, there exists $q_{1} \in \mathbb{N}$ such that, for all $q \geq q_{1}: \quad \tilde{y}_{i}<F\left(\tilde{x}_{i}, \tilde{z}_{i}, \varphi_{i}^{0}\left(z^{q}\right)\right)$, and either there exists $\bar{v}_{i}^{x, q}<\tilde{v}_{i}^{x}+\tilde{v}_{i}^{y} \quad$ solving $\quad g^{x}\left(\varphi_{i}^{0}\left(z^{q}\right), \bar{v}_{i}^{x, q}, \tilde{v}_{i}^{x}+\tilde{v}_{i}^{y}+\tilde{v}_{i}^{z}\right)=g^{x}\left(\varphi_{i}^{0}\left(z^{*}\right), \tilde{v}_{i}^{x}, \tilde{v}_{i}^{x}+\tilde{v}_{i}^{y}+\tilde{v}_{i}^{z}\right) \quad$ such $\quad$ that $g^{y}\left(\varphi_{i}^{0}\left(z^{q}\right), \tilde{v}_{i}^{x}+\tilde{v}_{i}^{y}-\bar{v}_{i}^{x, q}, \tilde{v}_{i}^{x}+\tilde{v}_{i}^{y}+\tilde{v}_{i}^{z}\right)>\tilde{y}_{i}$, if $\quad \tilde{v}_{i}^{x}+\tilde{v}_{i}^{y}=B$, or there exists $\bar{v}_{i}^{x, q} \quad$ solving $g^{x}\left(\varphi_{i}^{0}\left(z^{q}\right), \bar{v}_{i}^{x, q}, \tilde{v}_{i}^{x}+\tilde{v}_{i}^{y}+\tilde{v}_{i}^{z}\right)=g^{x}\left(\varphi_{i}^{0}\left(z^{*}\right), \tilde{v}_{i}^{x}, \tilde{v}_{i}^{x}+\tilde{v}_{i}^{y}+\tilde{v}_{i}^{z}\right) \quad$ and $\quad \bar{v}_{i}^{y, q} \quad$ solving $g^{y}\left(\varphi_{i}^{0}\left(z^{q}\right), \bar{v}_{i}^{y, q}, \tilde{v}_{i}^{x}+\tilde{v}_{i}^{y}+\tilde{v}_{i}^{z}\right)=g^{y}\left(\varphi_{i}^{0}\left(z^{*}\right), \tilde{v}_{i}^{y}, \tilde{v}_{i}^{x}+\tilde{v}_{i}^{y}+\tilde{v}_{i}^{z}\right)$ such that $\tilde{v}_{i}^{x, q}+\tilde{v}_{i}^{y, q}<B$, if $\tilde{v}_{i}^{x}+\tilde{v}_{i}^{y}<B$. We let then: $\left(\tilde{x}_{i}^{q}, \tilde{y}_{i}^{q}, \tilde{z}_{i}^{q}\right)=\left(\tilde{x}_{i}, \tilde{y}_{i}, \tilde{z}_{i}\right), \quad \tilde{v}_{i}^{x, q}=\bar{v}_{i}^{x, q}, \tilde{v}_{i}^{y, q}$ be either $=\tilde{v}_{i}^{x}+\tilde{v}_{i}^{y}-\bar{v}_{i}^{x, q}$ (if $\tilde{v}_{i}^{x}+\tilde{v}_{i}^{y}=B$ ) or $=\bar{v}_{i}^{y, q}$ (if $\left.\tilde{v}_{i}^{x}+\tilde{v}_{i}^{y}<B\right)$, and $\tilde{v}_{i}^{z, q}=\tilde{v}_{i}^{z}$, for all $q \geq \max \left\{q_{0}, q_{1}\right\} ;\left(\tilde{x}_{i}^{q}, \tilde{y}_{i}^{q}, \tilde{z}_{i}^{q}, \tilde{v}_{i}^{q}\right)$ be an arbitrary element 
of $A_{i}\left(\varphi_{i}^{0}\left(z^{q}\right), \omega_{i}\right)$ for all $q<\max \left\{q_{0}, q_{1}\right\}$. If $\tilde{y}_{i}=F\left(\tilde{x}_{i}, \tilde{z}_{i}, \varphi_{i}^{0}\left(z^{*}\right)\right)$, we have then $\tilde{v}_{i}^{x}+\tilde{v}_{i}^{y}=B$, $\tilde{v}_{i}^{z}=\left(g_{B}^{z}\right)^{-1}\left(\tilde{z}_{i}\right)$ and $\left(\tilde{x}_{i}, \tilde{y}_{i}, \tilde{z}_{i}\right)=g\left(\varphi_{i}^{0}\left(z^{*}\right), \tilde{v}_{i}\right)$ by construction of $F($ see Lemma 1), and we let: $\tilde{x}_{i}^{q}=\tilde{x}_{i}, \quad \tilde{z}_{i}^{q}=\tilde{z}_{i}, \quad \tilde{y}_{i}^{q}=F\left(\tilde{x}_{i}, \tilde{z}_{i}, \varphi_{i}^{0}\left(z^{q}\right)\right), \quad \tilde{v}_{i}^{x, q}$ solve $\tilde{x}_{i}=g^{x}\left(\varphi_{i}^{0}\left(z^{q}\right), \tilde{v}_{i}^{x, q}, B+\left(g_{B}^{z}\right)^{-1}\left(\tilde{z}_{i}\right)\right), \quad \tilde{v}_{i}^{y, q}$ solve $\tilde{y}_{i}^{q}=g^{y}\left(\varphi_{i}^{0}\left(z^{q}\right), \tilde{v}_{i}^{y, q}, B+\left(g_{B}^{z}\right)^{-1}\left(\tilde{z}_{i}\right)\right)$, and $\tilde{v}_{i}^{z, q}=\left(g_{B}^{z}\right)^{-1}\left(\tilde{z}_{i}\right)=\tilde{v}_{i}^{z}$ for all $q \geq q_{0} ;\left(\tilde{x}_{i}^{q}, \tilde{y}_{i}^{q}, \tilde{z}_{i}^{q}, \tilde{v}_{i}^{q}\right)$ be an arbitrary element of $A_{i}\left(\varphi_{i}^{0}\left(z^{q}\right), \omega_{i}\right)$ for all $q<q_{0}$. One verifies immediately that the sequence converges to $\left(\tilde{x}_{i}, \tilde{y}_{i}, \tilde{z}_{i}, \tilde{v}_{i}\right)$. We have $u\left(x_{i}^{q}, y_{i}^{q}\right) \geq u\left(\tilde{x}_{i}^{q}, \tilde{y}_{i}^{q}\right)$ for all $q$ by construction, so that $u\left(x_{i}^{*}, y_{i}^{*}\right) \geq u\left(\tilde{x}_{i}, \tilde{y}_{i}\right)$ by continuity of $u$. Therefore $\left(x_{i}^{*}, y_{i}^{*}, \bar{z}_{i}^{*}, v_{i}^{*}\right) \in \varphi_{i}^{C}\left(z^{*}\right)$, and the upper hemicontinuity of $\varphi_{i}^{C}$ is established.

\section{A-4: Existence of clear-sighted equilibrium}

The existence of a clear-sighted equilibrium is not warranted, in general, under Assumptions 1, 2 and 3. The appropriate tool for establishing existence is Debreu's social equilibrium existence theorem (1952), applied to the Nash non-cooperative equilibrium of the first stage of the clear-sighted game. The general condition for existence, implied by this theorem, which may fail to hold in the case of clear-sighted equilibrium is convex-valued reaction correspondences of hospitals. We show below that an equilibrium exists in an acceptable subset of the wider class of medical care systems considered in this article.

Lemma 4: If $(W, u, g, \omega)$ is convex, then $\varphi_{i}^{C}$ is convex-valued for all $i$.

Proof: We established in the proof of Lemma 3 that hospital $i$ 's first-stage program $\max \left\{u\left(x_{i}, y_{i}\right):\left(x_{i}, y_{i}, z_{i}, v_{i}\right) \in A_{i}\left(\varphi_{i}^{0}\left(\left(z_{n / i}, z_{i}\right)\right), \omega_{i}\right)\right\} \quad$ was equivalent to program $\max \left\{u\left(x_{i}, F\left(x_{i}, z_{i}, \varphi_{i}^{0}\left(\left(z_{n / i}, z_{i}\right)\right)\right)\right): 0 \leq z_{i} \leq \omega_{i}\right\}$ for any fixed $z_{n / i} \in\left\{z_{n / i} \in \mathbb{R}_{+}^{n-1}: z_{n / i} \leq \omega_{n / i}\right\}$, and yielded positive optimal graft production of hospital $i$ whenever $z_{n / i}=0$. The convexity assumption of 
Definition 5 is therefore exactly sufficient for the convexity of $\varphi_{i}^{C}(z)$ for all $i$ and all $z \in\left\{z \in \mathbb{R}_{+}^{n}: z \leq \omega\right\}$

Lemma 5: Let the medical care system $(W, u, g, \omega)$ be such that $\varphi_{i}^{C}$ is convex-valued for all $i$. Then there exists a clear-sighted equilibrium of $(W, u, g, \omega)$.

Proof: $\varphi^{0}$ is a continuous function $\left\{z \in \mathbb{R}_{+}^{n}: z \leq \omega\right\} \rightarrow \mathbb{R}_{+}^{n}$ by Lemma 2 , and $\varphi_{i}^{C}$ is an upper hemicontinuous correspondence $\left\{z \in \mathbb{R}_{+}^{n}: z \leq \omega\right\} \rightarrow \mathbb{R}_{+}^{6}$ for all $i$ by Lemma 3. Let the canonical projection $\left(x_{i}, y_{i}, z_{i}, v_{i}\right) \rightarrow z_{i}$ be denoted by $p r_{3} . \Phi^{C}: z \rightarrow\left(p r_{3}\left(\varphi_{1}^{C}(z)\right), \ldots, p r_{3}\left(\varphi_{n}^{C}(z)\right)\right)$ is an upper hemicontinuous, convex-valued correspondence $\left\{z \in \mathbb{R}_{+}^{n}: z \leq \omega\right\} \rightarrow\left\{z \in \mathbb{R}_{+}^{n}: z \leq \omega\right\}$. Set $\left\{z \in \mathbb{R}_{+}^{n}: z \leq \omega\right\}$ being non-empty compact and convex, $\Phi^{C}$ has a fixed point in $\left\{z \in \mathbb{R}_{+}^{n}: z \leq \omega\right\}$ by Kakutani's fixed point theorem, that is, there exists $z^{*} \in\left\{z \in \mathbb{R}_{+}^{n}: z \leq \omega\right\}$ such that $z^{*} \in \Phi^{C}\left(z^{*}\right)$. There exists, therefore, a state $\left(x^{*}, y^{*}, z^{*}, v^{*}, t^{*}\right)$ such that $t^{*}=\varphi_{i}^{0}\left(z^{*}\right)$ and $\left(x_{i}^{*}, y_{i}^{*}, z_{i}^{*}, v_{i}^{*}\right) \in \varphi_{i}^{C}\left(z^{*}\right)$ for all $i .\left(x^{*}, y^{*}, z^{*}, v^{*}, t^{*}\right)$ is an equilibrium of the clear-sighted game by construction

The medical care systems of Examples 1 and 2 are convex.

\section{A-5: Rotten kids and abundance}

Proof of Theorem 4: Part (i) of the Theorem is a simple consequence of Lemmas 4 and 5 (existence) and of Lemmas 2 and 3 (positivity). The qualitative aspects of parts (ii) and (iii) are supported by the discussion and the examples that follow Theorem 4 in subsection 4-2-2. Part (iii) is complemented by the following clear-cut statements, established below: If all programs $\left\{u\left(x_{i}, F\left(x_{i}, z_{i}, z_{i}\right)\right): z_{i} \leq \omega_{i}\right\}$ have a same solution $\left(x^{*}, z^{*}\right)$, then: rationing constraints are either all identical and binding or all nonbinding at $\left(x^{*}, z^{*}\right)$ in programs $\left\{u\left(x_{i}, F\left(x_{i}, z_{i}, z_{i}\right)\right): z_{i} \leq \omega_{i}\right\} ;\left(x^{*} \cdot e_{n}, F\left(x^{*}, z^{*}, z^{*}\right) \cdot e_{n}, z^{*} \cdot e_{n}\right)$ is a clearsighted equilibrium production combination, and agency's corresponding equilibrium transfer is 
$\varphi^{0}\left(z^{*} \cdot e_{n}\right)=z^{*} \cdot e_{n} ; \partial \varphi^{0}\left(z^{*}\right)$ is $=e_{n}$ if rationing constraints are all non-binding in programs $\left\{u\left(x_{i}, F\left(x_{i}, z_{i}, z_{i}\right)\right): z_{i} \leq \omega_{i}\right\}$, and $\ll e_{n}$ otherwise.

Let $\left(x^{*}, z^{*}\right)$ be a solution of $\left\{u\left(x_{i}, F\left(x_{i}, z_{i}, z_{i}\right)\right): z_{i} \leq \omega_{i}\right\}$, the same for all $i$, and let $F\left(x^{*}, z^{*}, z^{*}\right)$ be denoted by $y^{*}$. The boundary condition of Assumption 2 implies that production combination $\left(x^{*}, y^{*}, z^{*}\right)$ is $\gg 0$. The characterizing first-order conditions for this maximum read: $\frac{\partial_{1} u}{\partial_{2} u}=-\partial_{1} F$, $-\frac{\partial_{2} F}{\partial_{3} F} \leq 1$, and $\left(1+\frac{\partial_{2} F}{\partial_{3} F}\right) \cdot\left(\omega_{i}-z^{*}\right)=0$ for all $i$, where partial derivatives are evaluated at the optimum.

Identical $\left(x^{*}, y^{*}\right)$ imply that marginal social utilities $\partial_{i} W\left(u\left(x^{*}, y^{*}\right), \ldots, u\left(x^{*}, y^{*}\right)\right)$ are identical for all $i$ by the anonymity property of Assumption 3. Identical $\left(x^{*}, z^{*}\right)$ imply that hospitals have same $\partial F_{3}\left(\left(x^{*}, z^{*}, z^{*}\right)\right.$. The f.o.c. of Corollary 1 then imply that $\left(x^{*} \cdot e_{n}, y^{*} \cdot e_{n}, z^{*} \cdot e_{n}\right)$ is a socially optimal production combination of $(W, u, F, \omega)$.

If some rationing constraint is binding at $\left(x^{*}, z^{*}\right)$ in programs above, that is, if $-\frac{\partial_{2} F\left(x^{*}, z^{*}\right)}{\partial_{3} F\left(x^{*}, z^{*}\right)}<1$ and $z^{*}=\omega_{i}$ for some $i$, then, clearly, all rationing constraints are binding and identical, so that, in particular, all hospitals have the same endowment, $=z^{*}$. In other words, rationing constraints are either all identical and binding or all non-binding at $\left(x^{*}, z^{*}\right)$ in programs $\left\{u\left(x_{i}, F\left(x_{i}, z_{i}, z_{i}\right)\right): z_{i} \leq \omega_{i}\right\}$. Function $F$ being $C^{2}$ wherever it is defined in $\mathbb{R}_{++}^{3}$, and then such that $\partial_{3} F>0$, the implicit function theorem implies the existence of open neighbourhoods $U$ and $V$ of $z^{*}$ in $\mathbb{R}_{++}$and of a $C^{1}$ function $\psi: U \rightarrow V$ such that $\psi\left(z^{*}\right)=z^{*}, \quad$ and, for all $s \in U, \quad y^{*}=F\left(x^{*}, s, \psi(s)\right) \quad$ and $\partial \psi(s)=-\left(\partial F_{2}\left(x^{*}, s, \psi(s)\right) / \partial F_{3}\left(x^{*}, s, \psi(s)\right)\right)$. The f.o.c. of Lemma 2 then imply that the agency's second-stage optimal transfer policy identifies with function $\left(z_{1}, \ldots, z_{n}\right) \rightarrow\left(\psi\left(z_{1}\right), \ldots, \psi\left(z_{n}\right)\right)$ over $\left\{z \in U^{n}: z \leq \omega ; \exists \alpha \in \mathbb{R}_{++}\right.$such that $\left.z=\alpha . e_{n}\right\}$ (since all hospitals have same $\partial F_{3}\left(x_{i}^{*}, z_{i}, \psi\left(z_{i}\right)\right.$ ) for all $z$ in the latter set). In particular: $\varphi^{0}\left(z^{*}\right)=z^{*}$; and $\partial \varphi^{0}\left(z^{*}\right)$ is $=e_{n}$ if rationing constraints are all non- 
binding in programs $\left\{u\left(x_{i}, F\left(x_{i}, z_{i}, z_{i}\right)\right): z_{i} \leq \omega_{i}\right\}$, and $\ll e_{n}$ otherwise, that is, if rationing constraints are all binding and identical in these programs. The f.o.c. of Lemma 3 and the quasi-concavity properties of $\left(x_{i}, z_{i}\right) \rightarrow u\left(x_{i}, F\left(x_{i}, z_{i}, \varphi^{0}\left(z_{n / i}^{*}, z_{i}\right)\right)\right.$ ) (implied by the convexity of $(W, u, F, \omega)$ ) then imply that $\left(x^{*}, z^{*}\right)$ solves $\max \left\{u\left(x_{i}, F\left(x_{i}, z_{i}, \varphi_{i}^{0}\left(\left(z_{n i i}^{*}, z_{i}\right)\right)\right)\right): z_{i} \leq \omega_{i}\right\}$ for all $i$ (see Lemma 3), and therefore that $\left(x^{*} \cdot e_{n}, y^{*} \cdot e_{n}, z^{*} \cdot e_{n}\right)$ is a clear-sighted equilibrium production combination of $(W, u, F, \omega))$, and that $z^{*} \cdot e_{n}$ is the corresponding optimal transfer of the agency

\section{A-6: Monitored equilibrium}

Proof of Theorem 5: Let $\left(x^{*}, y^{*}, z^{*}, v^{*}, t^{*}\right)$ be a monitored equilibrium. Assumptions 1, 2 and 3 and the definition of monitored equilibrium clearly imply that $\left(x^{*}, y^{*}, z^{*}, v^{*}, t^{*}\right) \gg 0$. Hospital $i$ 's monitored opportunity set

$$
A_{i}^{M}\left(v_{i}^{z}, t_{i}^{*}\right)=\left\{\left(x_{i}, y_{i}, v_{i}^{x}, v_{i}^{y}\right) \in \mathbb{R}_{+}^{4}:\right.
$$

$x_{i} \leq g^{x}\left(t_{i}^{*}, v_{i}^{x}, v_{i}^{x}+v_{i}^{y}+v_{i}^{z}\right), y_{i} \leq g^{y}\left(t_{i}^{*}, v_{i}^{y}, v_{i}^{x}+v_{i}^{y}+v_{i}^{z}\right)$, and $\left.v_{i}^{x}+v_{i}^{y} \leq B\right\}$ is compact, convex, and has a non-empty intersection with $\mathbb{R}_{++}^{4}$. The Kuhn and Tucker first-order conditions are therefore necessary and sufficient for convex program $\max \left\{u\left(x_{i}, y_{i}\right):\left(x_{i}, y_{i}, v_{i}^{x}, v_{i}^{y}\right) \in A_{i}^{M}\left(v_{i}^{z}, t_{i}\right)\right\}$ at interior equilibrium solution $\left(x_{i}^{*}, y_{i}^{*}, v_{i}^{x^{*}}, v_{i}^{y^{*}}\right)$ by Arrow and Enthoven (1961: Theorems 1 and 2). They read: (i)

$v_{i}^{x^{*}}+v_{i}^{y^{*}}=B ; \quad$ (ii) $\quad\left(x_{i}^{*}, y_{i}^{*}, z_{i}^{*}\right)=g\left(t_{i}^{*}, v_{i}^{*}\right)$; and (iii) $\frac{\partial_{1} u\left(x_{i}^{*}, y_{i}^{*}\right)}{\partial_{2} u\left(x_{i}^{*}, y_{i}^{*}\right)}=\frac{\partial_{2} g^{y}\left(t_{i}^{*}, v_{i}^{y^{*}}, v_{i}^{x^{*}}+v_{i}^{y^{*}}+v_{i}^{z^{*}}\right)}{\partial_{2} g^{x}\left(t_{i}^{*}, v_{i}^{x^{*}}, v_{i}^{x^{*}}+v_{i}^{y^{*}}+v_{i}^{z^{*}}\right)}$. Or equivalently, by Lemma $1:$ (i) $v_{i}^{x^{*}}+v_{i}^{y^{*}}=B$; (ii) $y_{i}^{*}=F\left(x_{i}^{*}, z_{i}^{*}, t_{i}^{*}\right) ; \quad$ and $\frac{\partial_{1} u\left(x_{i}^{*}, y_{i}^{*}\right)}{\partial_{2} u\left(x_{i}^{*}, y_{i}^{*}\right)}=\partial_{1} F\left(x_{i}^{*}, z_{i}^{*}, t_{i}^{*}\right)$.

Suppose that $\left(x^{*}, y^{*}, z^{*}, v^{*}, t^{*}\right)$ is not a social optimum and let us derive a contradiction. There exists then, by Theorem 2, a social optimum $(x, y, z, v, t)$ such that $W\left(u\left(x_{1}, y_{1}\right), \ldots, u\left(x_{n}, y_{n}\right)\right)>W\left(u\left(x_{1}^{*}, y_{1}^{*}\right), \ldots, u\left(x_{n}^{*}, y_{n}^{*}\right)\right)$. But then $\left(x, y, v^{x}, v^{y}\right) \in \varphi^{M}\left(v^{z}, t\right)$ if $v_{i}^{x}+v_{i}^{y}=B$ for all $i$, by the characterizing f.o.c. of Theorem 2 and of paragraph above. This may be supposed 
without loss of generality for $(x, y, z, v, t)$ by the hypothesis of Theorem 8 . But $\left(z, v^{z}, t\right) \in A_{0}^{M}(\omega)$, as an immediate consequence of the definition of a social optimum. Therefore $\left(x^{*}, y^{*}, z^{*}, v^{*}, t^{*}\right)$ is not a monitored equilibrium, the wished contradiction.

\section{References}

Andreoni, J. (1988), Privately provided public goods in a large economy: The limits of altruism, Journal of Public Economics 35: 57-73.

Arrow, K.J. and A.C. Enthoven (1961), “Quasi-concave programming”, Econometrica 29: 779-800.

Becker, G.S. (1974), “A theory of social interactions”, Journal of Political Economy 82: 1063-1093.

Becker, G.S. (1981), A Treatise on the Family (Harvard University Press, Cambridge (MA) and London).

Bergstrom, T.C. (1989), "A fresh look at the rotten kid theorem - and other household mysteries", Journal of Political Economy 97: 1138-59.

Bergstrom, T.C. and R.C. Cornes (1983), "Independence of allocative efficiency from distribution in the theory of public goods", Econometrica 51: 1753-65.

Bergstrom, T.C. and H.A. Varian (1985a), “When do market games have transferable utility?”, Journal of Economic Theory 35:222-223.

Bergstrom, T.C. and H.A. Varian (1985b), "When are Nash equilibria independent of the distribution of agents' characteristics?", Review of Economic Studies 52: 715-18.

Chamberlin, J. (1974), "Provision of collective goods as a function of group size", American Political Science Review 68: 707-16.

Cornes, R.C. and E.C.D. Silva (1999), "Rotten kids, purity and perfection", Journal of Political Economy 107: 1034-40.

Cornes, R. and R. Hartley (2007), "Aggregative public good games," Journal of Public Economic Theory, 9, 201-19.

Debreu, G. (1952), “A social equilibrium existence theorem”, Proceedings of the National Academy of Sciences 38: 886-893. Reprinted in G. Debreu (1983), Mathematical Economics (Cambridge University Press, Cambridge) 50-58. 
Fries, T.L., Golding, E. and R. Romano (1991), "Private provision of public goods and the failure of the neutrality property in large finite economies", International Economic Review 32:147-57

Guttman, J. (1978), "Understanding collective action: Matching behaviour", American Economic Review 68: 251-55.

Guttman, J. (1987), “A non-Cournot model of voluntary collective action”, Econometrica 54: 1-19.

Mas-Colell, A. (1985), The Theory of General Equilibrium. A Differentiable Approach (Cambridge University Press, London).

Mercier Ythier, J. (2006), “The economic theory of gift-giving: Perfect substitutability of transfers and redistribution of wealth", Chap. 5 in S.-C. Kolm and J. Mercier Ythier eds., Handbook of the Economics of Giving, Altruism and Reciprocity (North-Holland, Amsterdam) 227-339.

Mercier Ythier (2007), "Regular distributive efficiency and the distributive liberal social contract". Working paper, BETA and IDEP.

Roth, Alvin E., Tayfun Sonmez, and M. Utku Unver (2005a), “A Kidney Exchange Clearinghouse in New England", American Economic Review 95: 376-380.

Roth, A. E., Tayfun Sonmez, and M. Utku Unver (2005b), "Pairwise Kidney Exchange", Journal of Economic Theory 125: 151-188.

Roth, A. E., Tayfun Sonmez, M. Utku Unver, Francis L. Delmonico, and Susan L. Saidman (2006), "Utilizing List Exchange and Undirected Good Samaritan Donation through 'Chain' Paired Kidney Exchanges", American Journal of Transplantation 6: 2694-2705.

Thorne, E.D. (1996), “The cost of procuring market-inalienable human organs”, Journal of Regulatory Economics 10: 191-200.

Thorne, E.D. (2000), “The common property nature of market-inalienability”, Chap. 2 in S.-C. Kolm, L.-A. Gérard-Varet and J. Mercier Ythier eds., The Economics of Reciprocity, Giving and Altruism (Macmillan, London) 47-77.

Thorne, E.D. (2006), "The economics of organ transplantation", Chap. 20 in S.-C. Kolm and J. Mercier Ythier eds., Handbook of the Economics of Giving, Altruism and Reciprocity (North-Holland, Amsterdam) 1335-1370. 